\title{
RFID CLASSROOM MANAGEMENT SYSTEM
}

\author{
A Thesis \\ Presented to \\ the Faculty of California Polytechnic State University \\ San Luis Obispo
}

\author{
In Partial Fulfillment \\ of the Requirements for the Degree \\ Master of Science in Industrial Engineering
}

by

Andrew W. Wright

June 2011 
(C) 2011

Andrew W. Wright

ALL RIGHTS RESERVED 
TITLE:

AUTHOR:

DATE SUBMITTED:

COMMITTEE CHAIR:

COMMITTEE MEMBER:

COMMITTEE MEMBER:

\section{RFID Classroom Management}

Andrew W. Wright

June 10, 2011
Dr. Liz Schlemer, Associate Professor

Dr. Lily Laiho, Assistance Professor 


\section{ABSTRACT \\ RFID Classroom Management System \\ Andrew W. Wright}

Professors who manage large classes are unrealistically expected to grade each student fairly and accurately. Even with all of the technological advancements that have occurred in the past thirty years, very little progress has been made in classroom management, and as a result, professors are not equipped with enough tools to successfully manage large class sizes. Because radio frequency identification (RFID) technology is making its way into student issued identification cards, there is an opportunity to use it as a tool to aid professors in the classroom. The focus of this paper is to discover the most effective system that can be implemented as a classroom management instrument. Through multi criteria analysis, several different infrastructures are examined and compared to determine the best alternative. The result of an effective system leads to a reduction in time spent taking attendance, an increase in student performance, an increase in the fairness and accuracy of recording classroom participation, and an enhanced professor-student relationship.

Keywords: Radio Frequency Identification (RFID), Localization, Classroom Management 


\section{ACKNOWLEGEMENTS}

I would like to thank Dr. Tali Freed for providing the time and resources

necessary to complete this research, and Tyler Peabody for his help and effort in the user interface portion of this project. 


\section{TABLE OF CONTENTS}

LIST OF TABLES

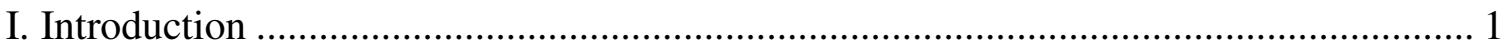

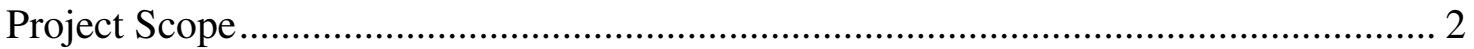

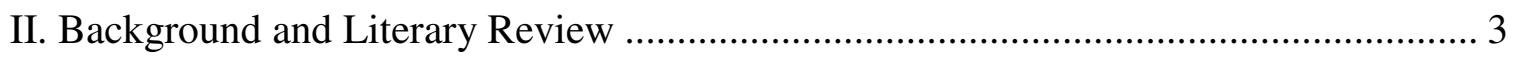

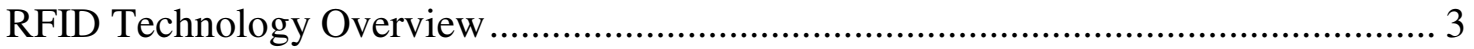

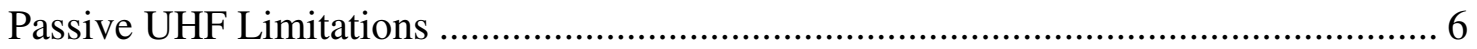

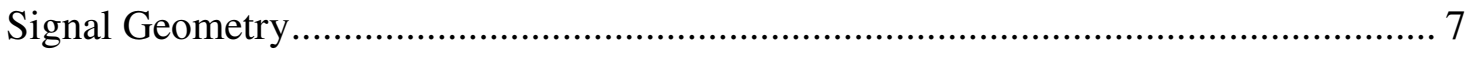

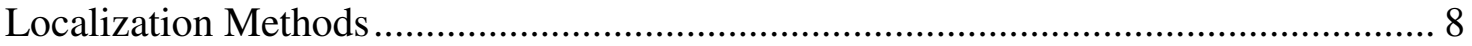

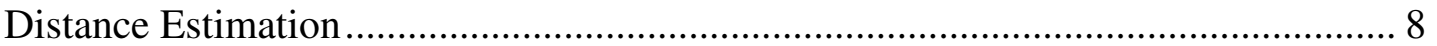

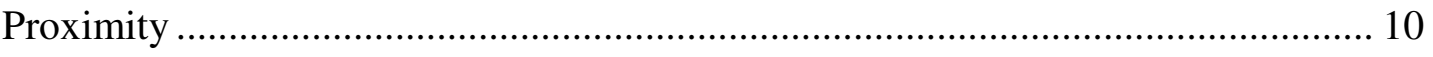

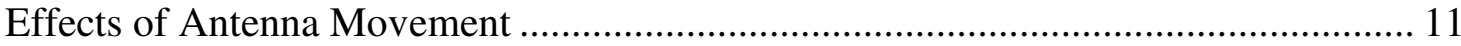

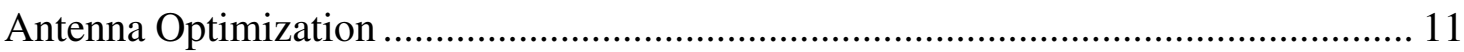

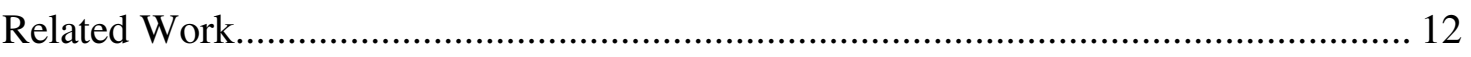

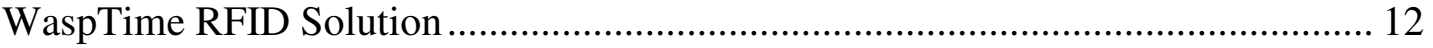

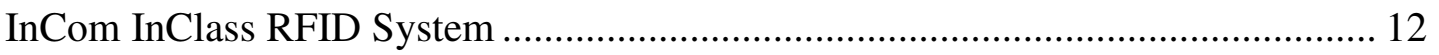

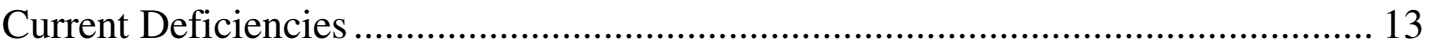

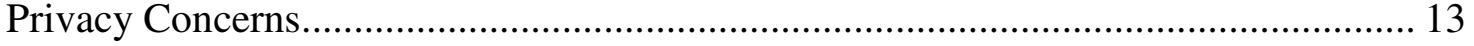

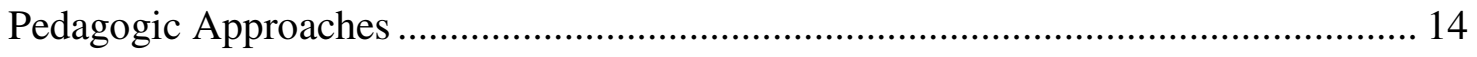

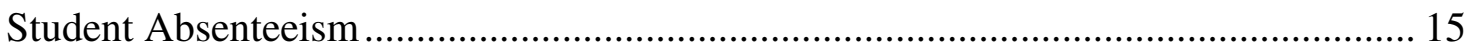

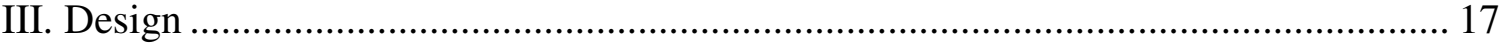

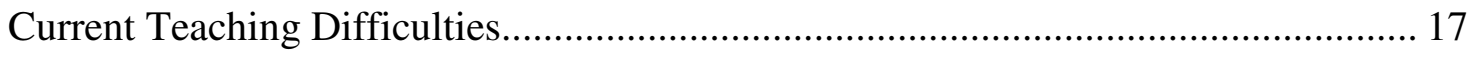

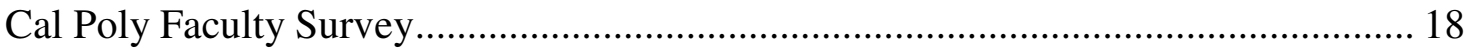

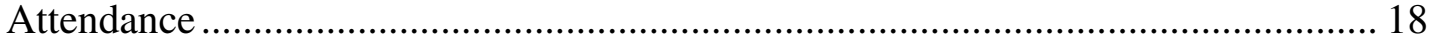

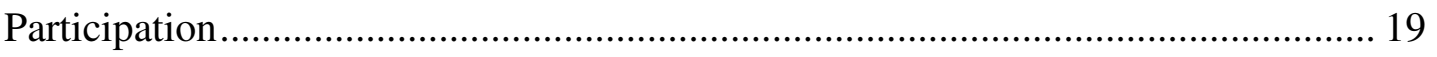

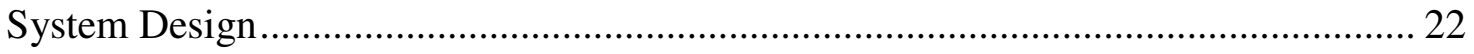

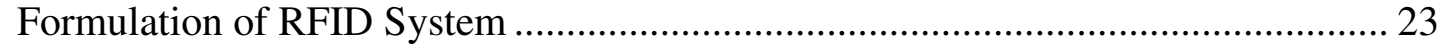

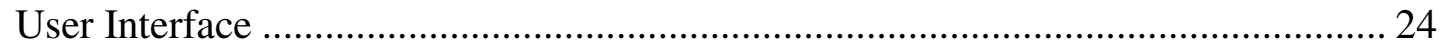

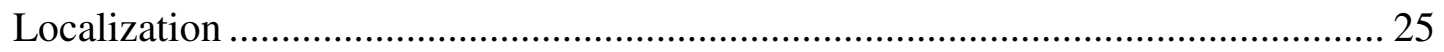




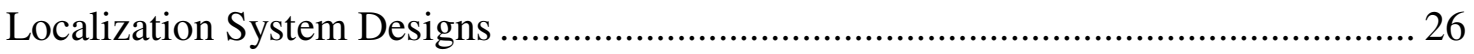

Ceiling Corner Four-Antenna System ................................................................. 26

Overhead Multiple-Antenna System .................................................................... 28

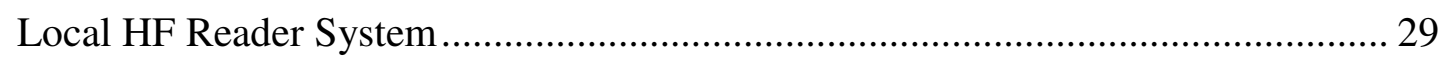

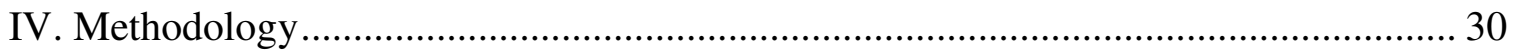

Ceiling Corner Four-Antenna System.................................................................... 30

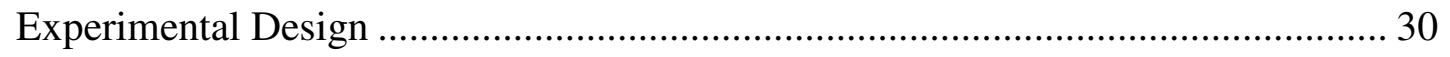

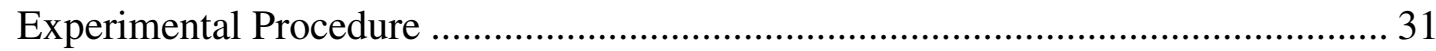

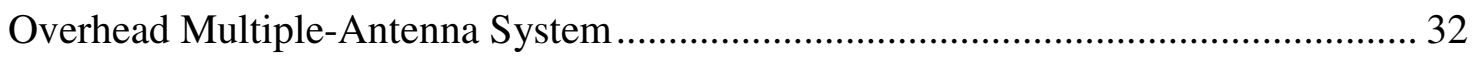

Experimental Design ........................................................................................ 33

Experimental Procedure ................................................................................... 35

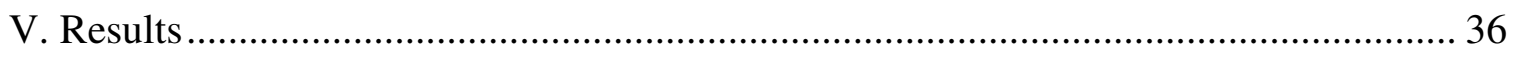

Ceiling Corner Four-Antenna System................................................................. 36

Overhead Multiple-Antenna System ........................................................................ 37

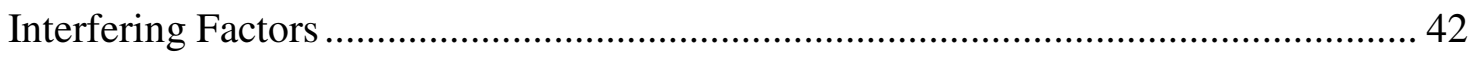

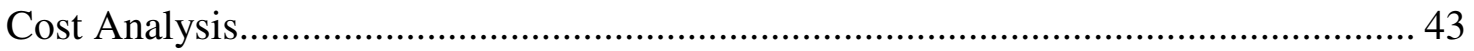

VI. Conclusions and Further Analysis ........................................................................... 46

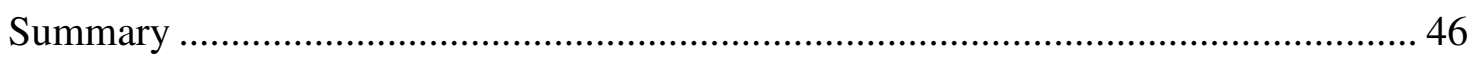

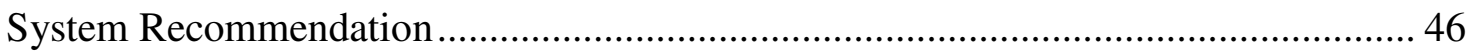

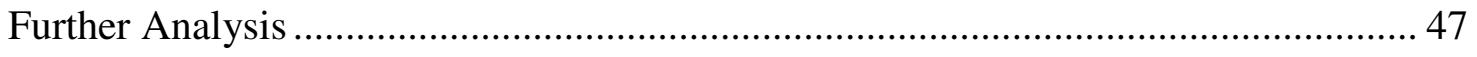

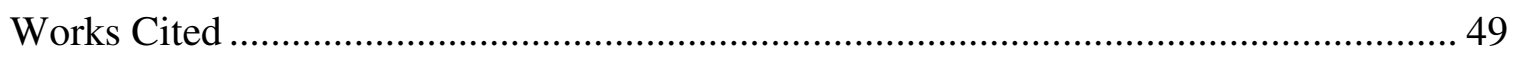

Appendix A: Statistical Analyses of Results ................................................................. 52

Significance of Tag Orientation .......................................................................... 52

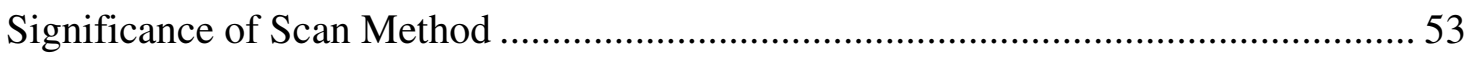

Significance of Light Fixture Interference .............................................................. 54

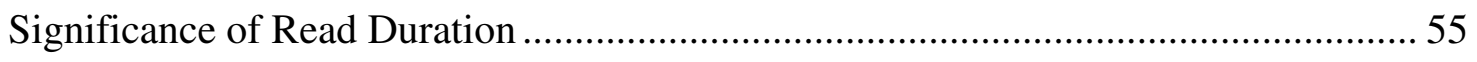

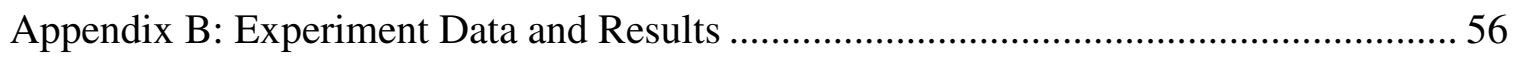

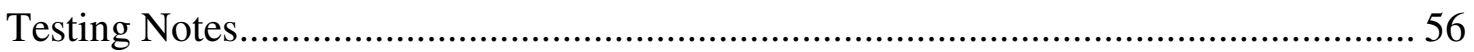

Appendix C: Program Code............................................................................... 57

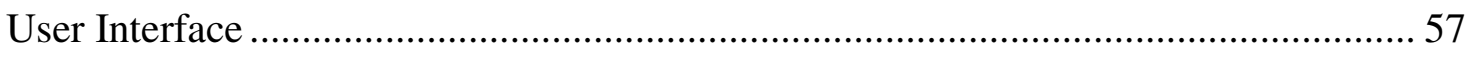

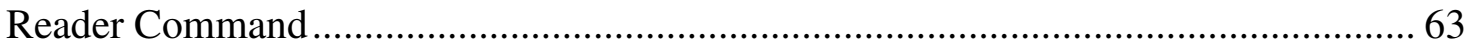




\section{LIST OF TABLES}

Table $\quad$ Page

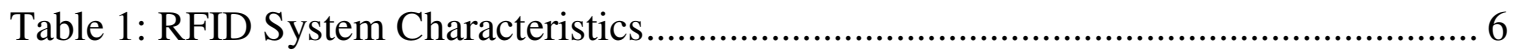

Table 2: Results of read distance relative to presence of water ...................................... 7

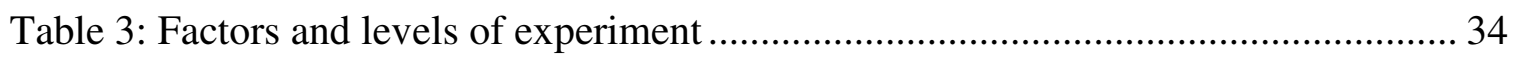

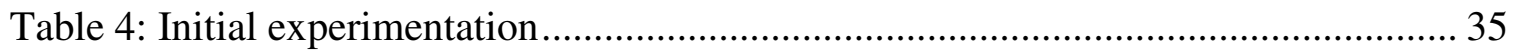

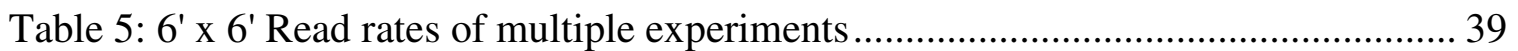

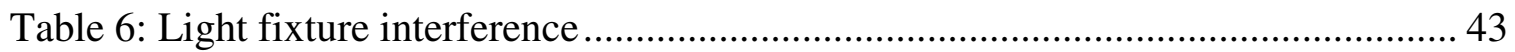

Table 7: Cost per classroom of four antenna UHF RFID system ................................ 44

Table 8: Cost per classroom of eight reader HF RFID system ................................... 44 


\section{LIST OF FIGURES}

Figure $\quad$ Page

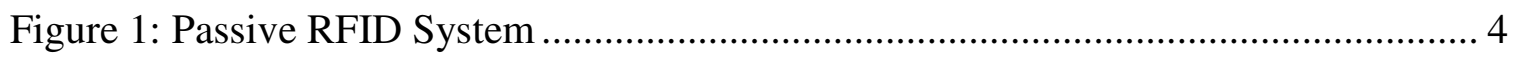

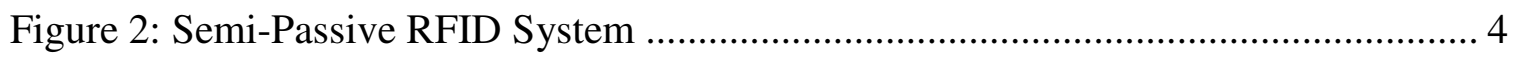

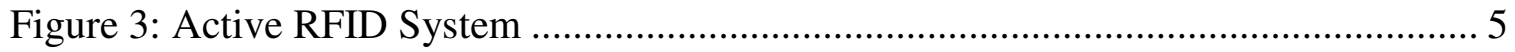

Figure 4: Beam approximation of a directional antenna.......................................... 8

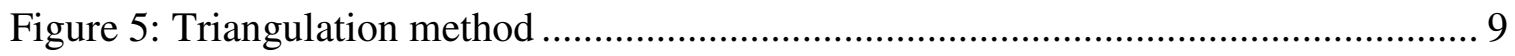

Figure 6: Trilateration in two dimensional space .............................................. 10

Figure 7: Survey attendance results - current system (above) v. fully automated system

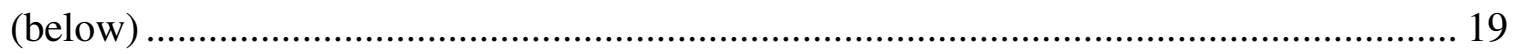

Figure 8: Survey participation results - reasons for not recording participation ............. 21

Figure 9: Survey participation results - current system (above) v. easier system (below)

Figure 10: Screenshot of prototype user interface .................................................. 24

Figure 11: Proximity Ceiling Corner Four-Antenna System ...................................... 27

Figure 12: Proximity Overhead Multiple-Antenna Design .......................................... 29

Figure 13: 12' x 16' experiment room grid (left); PVC, string, and RFID tag positions

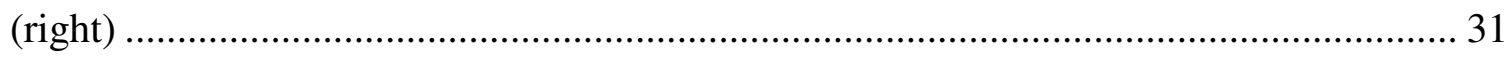

Figure 14: Setup of Control Room with Suspended Tags ........................................... 32

Figure 15: Tag board construction (left) and initial testing layout (right) ...................... 34

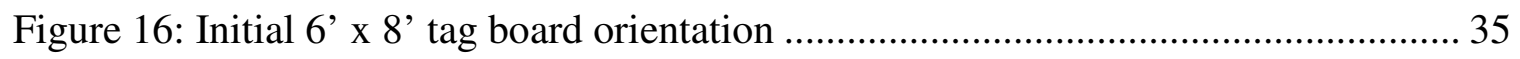

Figure 17: Perspective read rates of Ceiling Corner Four-Antenna System..................... 37

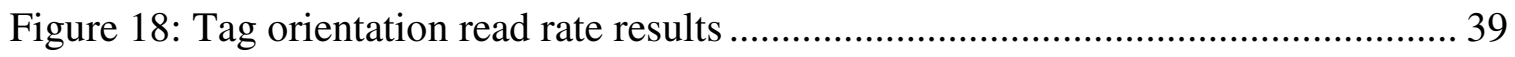


Figure 19: Positioning of tag boards in secondary testing ....................................... 40

Figure 20: Extended read range results.............................................................. 41

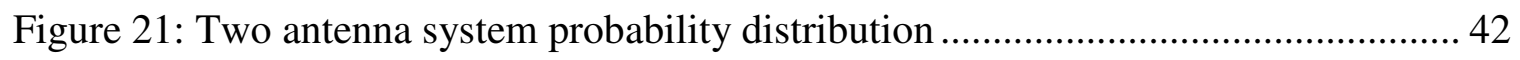




\section{Introduction}

Large class sizes at universities make it difficult for professors to efficiently take attendance, fairly record student participation, and learn students' names. In shorter classes, professors cannot afford to waste precious time with the roll call process of reading off a name, then scanning the classroom for a response. Additionally, professors in these situations struggle with keeping track of which students are actively participating in discussion. A majority of professors attempt to remember who was actively participating during class afterwards, which can lead to grading mistakes. This happens simply because it is difficult to associate every student with a name, and takes too long to make a note of an individual's participation credit on such a long roster.

Many universities are beginning to update their standard issue student identification cards to be equipped with radio frequency identification (RFID) tags. This decision opens up a window to implement RFID systems in classrooms as a management tool to solve many of the problems professors face. An RFID system which locates student's identification cards within a classroom can be used to provide a virtual layout of each student's position on a touchscreen device to a professor. Representing student positions in the classroom with photographs and names supplies the professor with a tool to better individualize the student learning experience, grade participation fairly, and learn students' names to establish essential relationships. The purpose of the paper is to not only determine the most effective system to locate students in a classroom, but construct a prototype user interface that an instructor can efficiently utilize as a classroom tool. Information delivered by this system can be used to take attendance, learn students' 
names faster, and even manipulated to greatly increase the ease of recording student participation.

\section{Project Scope}

This report will seek to develop a system capable of being implemented as a classroom management tool. Although much emphasis will lie on developing a working RFID localization method to be used inside classrooms, there will be a focus on designing a basic prototype of a user interface system. A clear path will be blazed to reveal the steps necessary to perfect the system for full implementation and possible commercial use. 


\section{Background and Literary Review}

The last decade has shown an enormous increase in RFID applications and techniques. Fueled by large retail corporations requiring suppliers to use RFID systems in their supply chains (Weinstein, 2005), the technology is continuously uncovering new purposes and functions. No longer is RFID just an improvement to bar codes; it is being used to track livestock, indicate when food has gone bad, and make quick purchases with cell phones.

\section{RFID Technology Overview}

The fundamental function of a radio frequency identification system is to use wireless communication to identify an object. RFID technology's primary advantage is that it does not require a line of site to detect an object, and can therefore simultaneously identify multiple objects within an area. A typical RFID system consists of a reader, antenna, tag, and computer or server. The basic components of a tag are a printed metal inlay, which serves as a small antenna, and a microchip, which contains the unique information of that tag. The communication process begins when the reader, acting as an interrogator, transmits a signal at a specific frequency through its antenna. If a tag is within the range of this antenna, it broadcasts a signal containing a unique identification number back to the reader, alerting it the tag is in the vicinity. Generally, a tag is attached to an object of interest, and a computer database is used to link the tag identification number to the specific object be tracked or located. 
There are three main types of RFID systems: passive, semi-passive, and active which are illustrated in Figure 1, Figure 2, and Figure 3, respectively. In a passive system, the transmitted signal from the reader powers the tag's integrated circuit (IC) reaches a tag, the tag reflects the signal in the form of backscatter. This reflected signal is modulated by the tag to transmit its unique identifier.

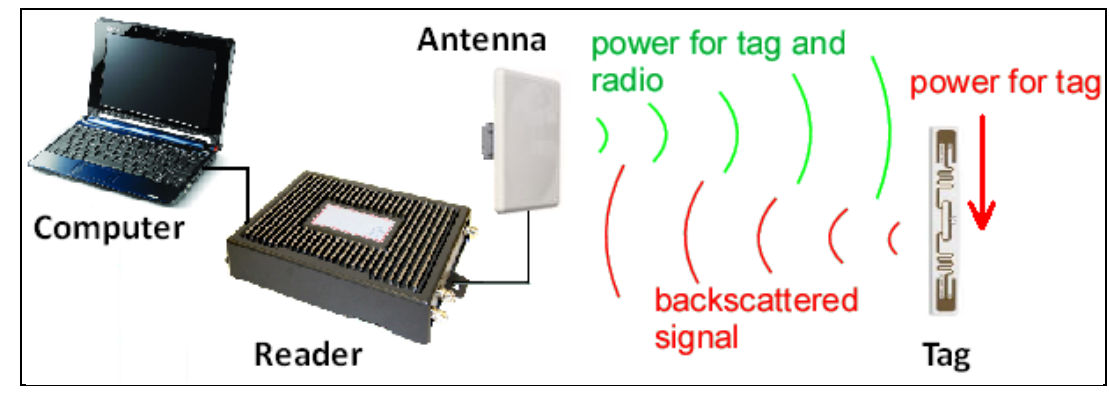

Source: Dobkin, 2007

Figure 1: Passive RFID System

Similarly, a semi-passive system reflects the signal from the antenna, but the backscatter signal it sends back is much more powerful because the tag is attached to a small battery. This battery provides the power to transmit the signal back to the reader, increasing its range.

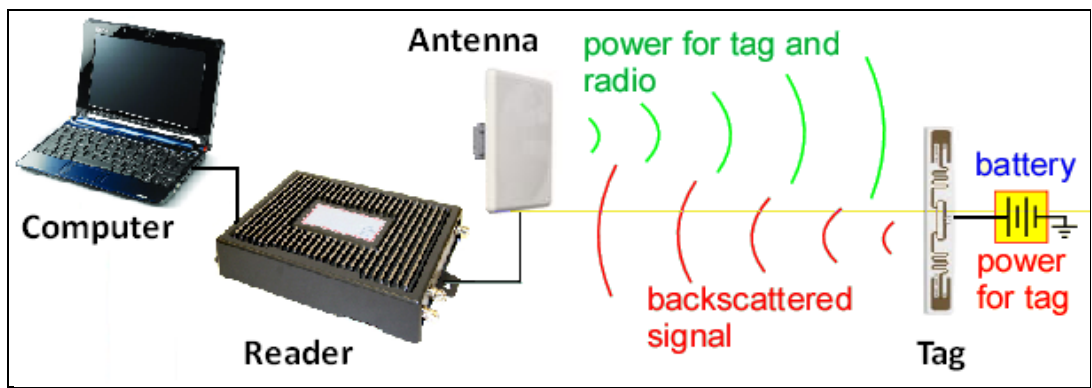

Source: Dobkin, 2007

Figure 2: Semi-Passive RFID System 
An active system is battery powered, but unlike the other two systems, it transmits its signal continuously, and does not need to be activated by the signal from the reader. Thus, the reader only needs to "listen" for the active tags signal (Want, 2006).

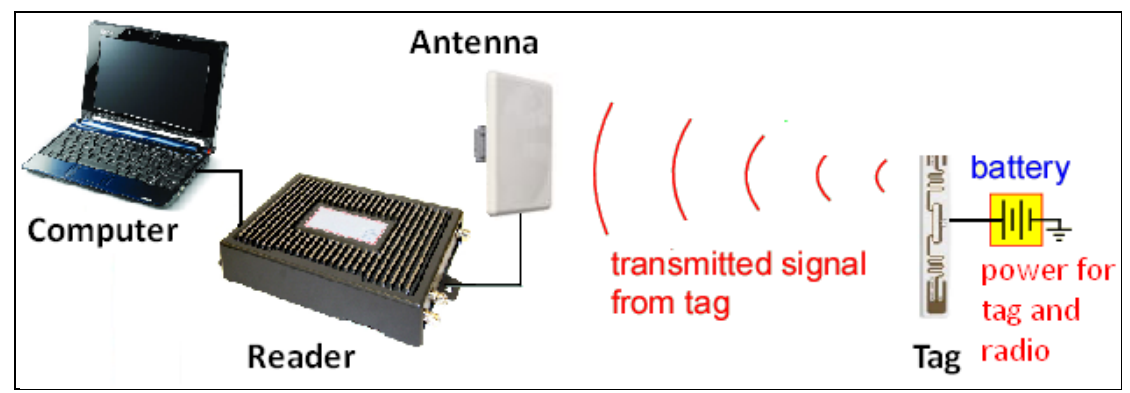

Source: Dobkin, 2007

Figure 3: Active RFID System

Within passive RFID systems, there are three frequency bands available: Low Frequency (LF), High Frequency (HF), and Ultra High Frequency (UHF).

Table 1 shows that when compared to semi-passive and active systems, these three passive systems have much shorter read ranges, ranging from $1 \mathrm{~cm}$ to $10 \mathrm{~m}$, because their backscattered signal is not battery powered (Weinstein, 2005). While this is a disadvantage of passive systems, the pivotal distinction in the comparison of passive and active systems for the purpose of a classroom management system is the price of individual tags. An active tag is generally a magnitude of one hundred times more expensive than a passive tag, usually costing around $\$ 15$, compared to a passive tag for $\$ .15$. 
Table 1: RFID System Characteristics

\begin{tabular}{|l|l|l|l|}
\hline RFID System & Frequency & Read Range & Price/Tag \\
\hline Passive LF & $125 / 134 \mathrm{KHz}$ & $1-3 \mathrm{~cm}$ & \\
\hline Passive HF & $13.56 \mathrm{MHz}$ & $1-10 \mathrm{~cm}$ & $\$ .10-\$ .25$ \\
\hline Passive UHF & $902-928 \mathrm{MHz}$ & $10 \mathrm{~m}$ & \\
\hline Semi-Passive & Varies & $100 \mathrm{~m}$ & $\$ 2-\$ 20$ \\
\hline Active & Varies & $100 \mathrm{~m}+$ & $\$ 20-\$ 100$ \\
\hline
\end{tabular}

\section{Passive UHF Limitations}

Although a passive UHF system has many advantages, there are factors to be considered when designing and implementing such a system. Primarily, there are two interfering factors specific to the $902-928 \mathrm{MHz}$ frequency band: signal absorption from water, and signal reflection from metal (Dobkin, 2007).

Radiated fields can be absorbed by many different materials in buildings and the environment, but the absorption rate of these waves into water increases with higher frequencies, and consequently has a much larger impact on the UHF band than HF or LF. This results in a decrease of readability of tags which are in the vicinity of water, illustrated by the Georgia Tech study shown below in Table 2. Because humans are made up of approximately $70 \%$ water, their presence in an RFID system must be addressed in the design. 
Table 2: Results of read distance relative to presence of water

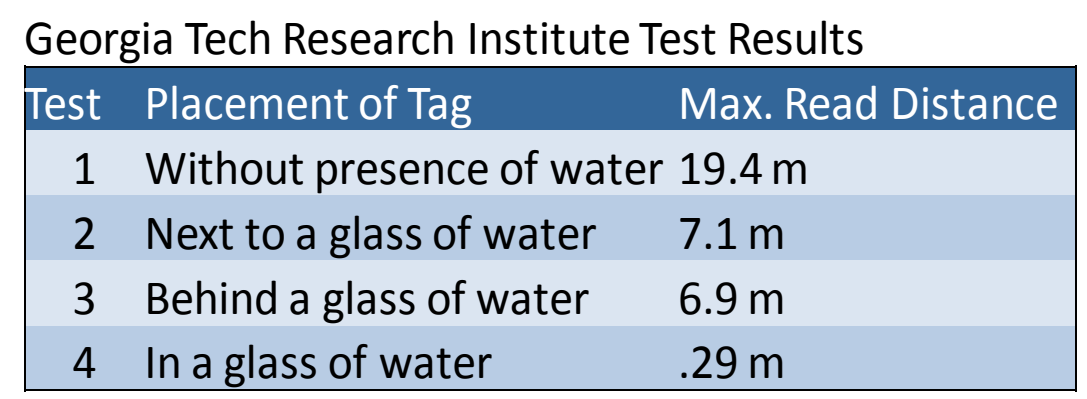

Source: Banks \& Thompson for AVISIAN Publications

The density of most metals is so great that they will interfere with an electric field by reflecting it off of the metal surface. The presence of metal objects in the field of an antenna will lead to obstruction of the signal, and therefore must be considered in the design phase of an RFID system. Additionally, typical tags cannot be placed directly on metal surfaces because the specific geometry of the tag's metal antenna will be interfered with.

\section{Signal Geometry}

It is important to understand the geometry of an antenna field when designing a system. Figure 4 illustrates the difference between the actual and approximated shape of the wave propagation. This approximated shape is assumed in the methodology and design throughout this report. 


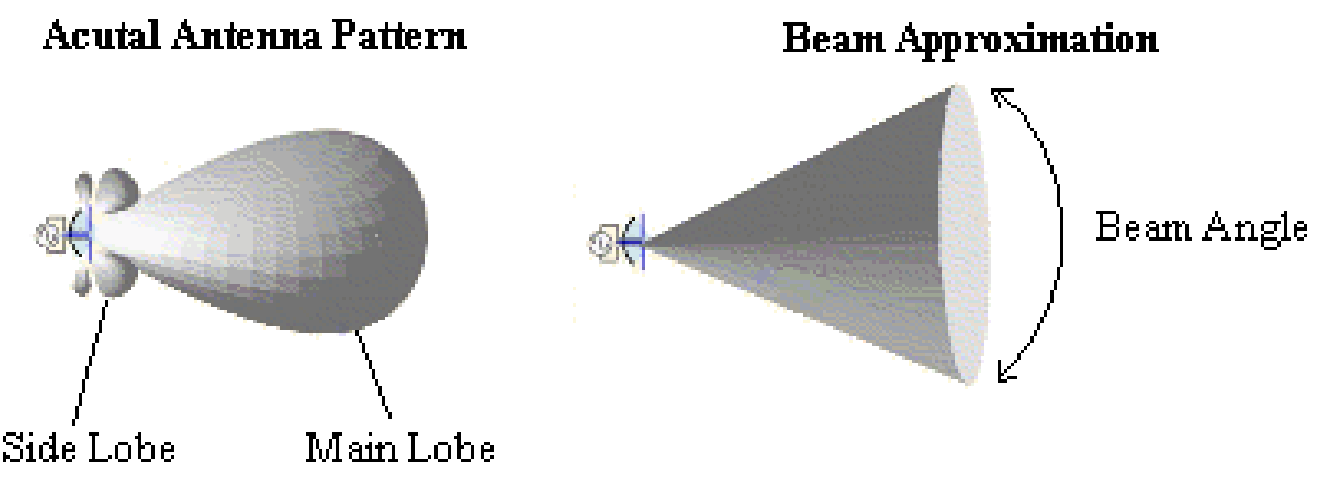

Source: Dobkin

Figure 4: Beam approximation of a directional antenna

\section{Localization Methods}

Due to the many interfering factors found in indoor environments, such as absorption and reflection, it is very difficult to precisely measure a signal. Thus, many localization methods have been presented in literature. However, most of these methods fall into the two categories of distance estimation, and proximity (Bouet, 2009).

\section{Distance Estimation}

Distance estimation locates a tag by using various properties of triangles. The two prevailing methods are triangulation and trilateration.

The triangulation method, shown in Figure 5, uses the Angle of Arrival (AOA) of at least two reference points. The intersection of these two angles defines the location of the tag. While a two dimension method is illustrated, multiple reference points can locate a tag in three dimensional space. 


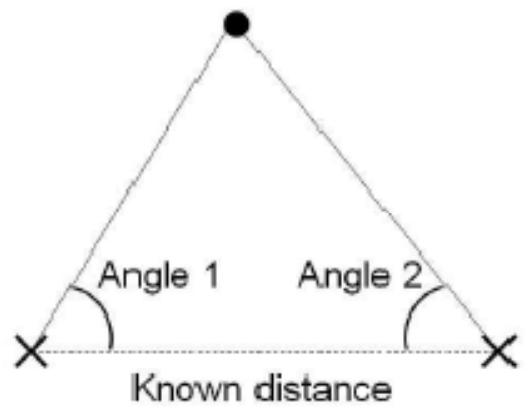

Source: Bouet, Matthew. RFID Tags: Positioning Principles and Localization Techniques. Laboratoire d'Informatique de Paris 6 Universit'e Pierre et Marie Curie, 2009.

Figure 5: Triangulation method

Trilateration is a locating technique used to find the two dimensional or three dimensional location of an object in space. Using the same basic principles as GPS, it determines the distance, but not direction, an object is from a reference point. In two dimensional space, this creates a circular line around the reference point which represents all the possible locations of the object. By using three or more reference points, the possible locations of the object are narrowed down to the intersection of all three of the reference circles, which effectively pinpoint its location. This basic principle is shown in Figure 6. 


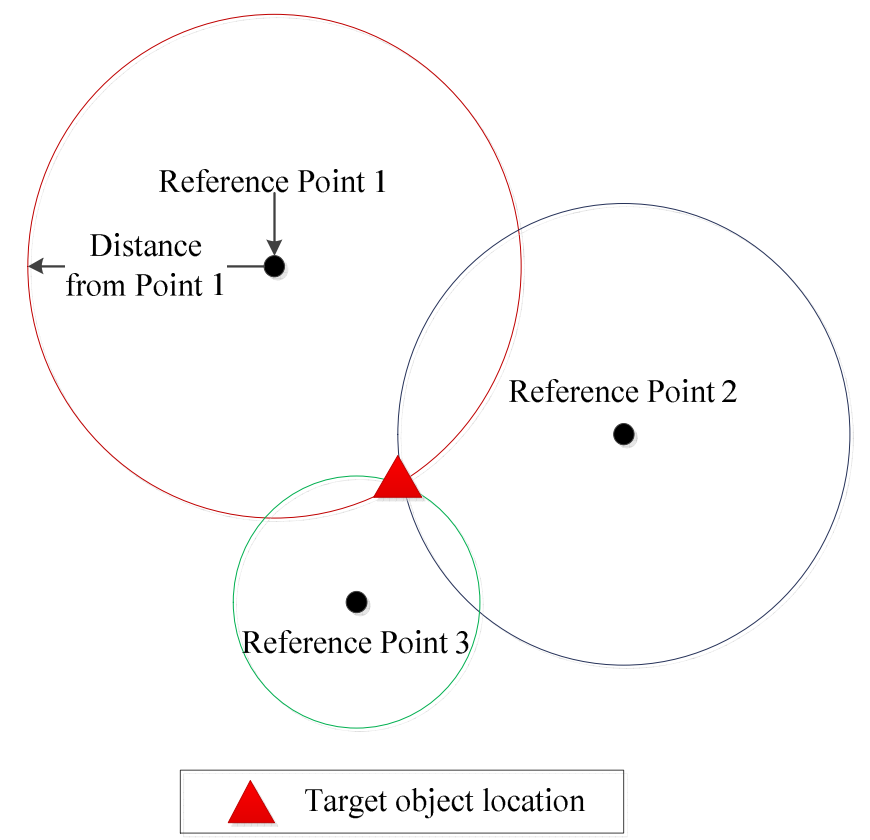

Figure 6: Trilateration in two dimensional space

Distance estimation methods provide relatively accurate localization, but require a considerable amount of information in regards to the metrics required (Sanpechuda et al., 2008). Many systems and algorithms that are able to locate tags have been recently developed, including SpotOn, which uses Received Signal Strength (RSS) (Hightower et al., 2000). Bouet introduces many systems using different metrics, including: Time of Arrival (TOA), Time Difference of Arrival (TDA), or as shown by Hekimian-Williams, 2010, using Received Signal Phase (RSP). These systems utilize a very high granularity, and are therefore highly accurate. However, in most commercial systems, these metrics are not available, and would require customized algorithms and systems to implement.

\section{Proximity}

Proximity systems rely on dense antennae coverage to locate tags. If a tag is read by an antenna, it can be assumed that it is within that antennas field. If it is also read by 
an adjacent antenna, it can be assumed that the tag is located in the overlap of the two antennae fields (Zhou et al., 2008). In addition, signal strength can be used to more accurately determine the location of the tag. Systems using the proximity method have been used to cover large areas where a high level of accuracy is not necessary, such as a construction site (Song et al., 2007).

Although the proximity method is limited in accuracy by the size of each antenna's field, and the density of antennae, it is extremely simple to implement with most commercial systems, and does not require the advanced metrics that distance estimation relies on. Consequently, this localization method is much more applicable to a classroom setting.

\section{Effects of Antenna Movement}

The previous methods of locating tags have all been stationary systems. It must be noted that there is a possible advantage of recording passive UHF tags while the antenna is moving. This is shown through the implementation of RFID antennas onto moving forklifts, and the associated increases in tag read rates (Freed and Krist, 2009).

\section{Antenna Optimization}

Covering an area accurately with the minimal number of antennas is important when they cost approximately $\$ 100$ each. In addition, knowing where to position antennas and the location of the resultant radiated signals is extremely important to the design and installation of RFID systems. Thus, there are algorithms and programs which calculate these parameters. Bryce Taylor, a Cal Poly student, developed such a program 
for his thesis. As Taylor states, that program, RFIDMIN, "can be used in conjunction with LP software or an algorithm to determine the minimum number and location of RFID antennas, such that any given 2D and 3D space is covered by at least one antenna" (Taylor 2008; Freed and Taylor, 2008).

\section{Related Work}

Many systems have been developed and tested that are able to register attendance of events or specific rooms using simple RFID configurations. Below is a brief overview of two systems which could be applied to an educational setting.

\section{WaspTime RFID Solution}

This much more labor-intensive system uses a high frequency (HF) reader which requires individuals to swipe their RFID cards or badges within three inches of the device to record the time and owner of the badge. This system is designed as an employee time keeping system and is not designed for schools specifically (Wasp Barcode, 2010).

\section{InCom InClass RFID System}

This system records attendance by using an overhead antenna at every classroom's doorway to automatically detect when students wearing RFID badges enter or exit the room. Because it uses a passive UHF RFID system, students are not required to slow down and swipe their badge or change their normal habits of entering a room. It is the only school attendance system available which requires no contact to operate (InCom Corporation, 2010). 


\section{Current Deficiencies}

Although each of these systems is able to take attendance and detect if a tag is within a certain room, neither has the ability to locate a tag to a specific position inside the room. In addition, there are no systems available that provide an interface for professors to record students' participation, or learn students' names.

\section{Privacy Concerns}

As RFID technology becomes more prevalent in everyday life, there are growing concerns about its security and tracking ability. An excellent example is when a small RFID company named InCom tested their attendance taking system in a local California school district. The system required students to where a lanyard holding an identification card with their photo, name, and embedded RFID tag. The company was surprised when after one week of implementing the system parents became outraged that their child was being tracked at school (O'Connor, 2005). Even though the technology only took attendance inside specific rooms, many parents felt their child's civil liberties were being violated. InCom quickly pulled the plug on the operation, but not after exposing a critical issue concerning RFID implementation.

While there are definitely lines that never should be crossed concerning a person's privacy, the environment that a classroom management tool operates in differs drastically from other location applications. Northern Arizona University (NAU) is currently defending RFID attendance taking systems it plans to implement by stating that "teachers are already asked to collect attendance manually_ and to incorporate attendance data into 
students' grades - and that using the RFID cards to automate the system would simply enable those who teach large classes to save time" (O'Connor, 2010).

A student does not have a choice to be recorded for attendance with or without RFID, so it is not justified to discriminate against the technology. If an RFID system simply makes a current accepted process more efficient, and does not intrude upon the privacy or liberties of an individual, it should be allowed to flourish. However, in an effort to reduce privacy concerns, the system developed in this report will provide students with an alternative option to being automatically scanned.

\section{Pedagogic Approaches}

While there is a multitude of different views concerning education, and specifically what the relationship between student and teacher should be, one thing is true: personal relationships with students ensure that students feel respected as individuals (Cole, 1999).

This respect helps to build student confidence and autonomy, two characteristics students struggle with most during their educational experiences (Mensch et al., 2002). While personal relationships do not hinge on knowing one another's name, it can be a large hindrance in the growth and establishment of a good rapport. Learning a person's name is most often a barrier of entry into a relationship. Additionally, knowing an individual student allows professors to recognize learning deficiencies, curtail the education to improve them, and consequently enhance the effectiveness of instruction. For professors, learning names can be extremely difficult with so many students to cover, and they are given very little to assist them in the process. 


\section{Student Absenteeism}

The effects of attendance on class performance has been widely researched and studied. Arulampalam et al. demonstrated that although the complete effect of student absenteeism varies with ability, higher rates of absenteeism have a direct correlation to poorer class performance (2007). Moore reinforces the claim by also finding an increase in student performance in a freshman level biology course in which the importance of attendance was emphasized, compared to one where it was not (2003). Devadoss et al. published a study which provided strong empirical evidence of the positive influence of attendance on performance (1996). In addition, he discovered a factor that lead to an increase in students' attendance. He states:

Whether or not the instructor "required" class attendance strongly influences students' behavior. All else being equal, an attendance requirement resulted in a $12.7 \%$ higher attendance rate. This supports the notion that "encouraging" students to come to classes - either through penalizing them by reducing scores or by requiring written make-up of missed class materials - seems to increase class attendance. (1996)

Among Devadoss's recommendations to increase attendance and performance is for professors to allocate a certain percentage of the total grade for attendance (1996).

Both of the claims of attendance's effects on performance and the motivational factors which lead to increased attendance are further substantiated by White as he remarks that in his agricultural policy class, requiring and rewarding attendance improved 
class attendance, and that student absenteeism resulted in lower grades (1992).

Therefore, it can be concluded that requiring attendance leads to an increase in student performance. 


\section{Design}

This chapter will describe the overall approach and steps taken to defining and solving the inherent deficiencies of modern classroom management. It begins with identifying the current problems in today's classrooms, and the associated needs of university professors. Characteristics of an optimal system are developed using this information, and are used to determine the design solution. The basic structure of this solution is presented, along with several configurations that require testing and evaluation.

\section{Current Teaching Difficulties}

California Polytechnic State University (Cal Poly) has an average class size of 35 ("Cal Poly, San Luis Obispo", 2011), which is considered small among large public universities. Professors at Cal Poly, and many other universities, face several challenges when trying to manage large class sizes. The first difficulty, and the root cause of others, is simply identifying each student by name. Although some professors are capable of learning every student's name, many find the task too difficult when faced with large class sizes, and at minimum it takes some time before every student becomes recognizable. This challenge is compounded when teachers attempt to grade students on their in-class participation. If a professor cannot recognize every student in the class, then he or she cannot accurately record participation.

The following sections provide an in depth analysis of two classroom management challenges: 
- Recording attendance

- Recording in-class participation

\section{Cal Poly Faculty Survey}

A survey of $100 \mathrm{Cal}$ Poly professors was administered to assess teaching practices and difficulties related to attendance taking and participation grading, as well as to evaluate the potential need for a classroom management system.

Attendance

As previously discussed, lower attendance has been shown to cause poorer class performance, which agrees with intuitive logic. Although professors have different opinions on including attendance in grading schemes, many have argued that because it is a university's responsibility to provide the best education it can, students should be encouraged to attend class. Giving students credit for attending class has been previously shown to increase the attendance rate, as well as the class performance. Unfortunately, recording attendance can be extremely tedious and time consuming in large classes. The survey asked Cal Poly professors how often they currently take attendance, as well as how often they would take attendance with a hypothetical fully automated system. The results are illustrated in Figure 7, and show a projected increase in attendance recording which clearly proves that professors desire a simple and automated attendance taking system. 


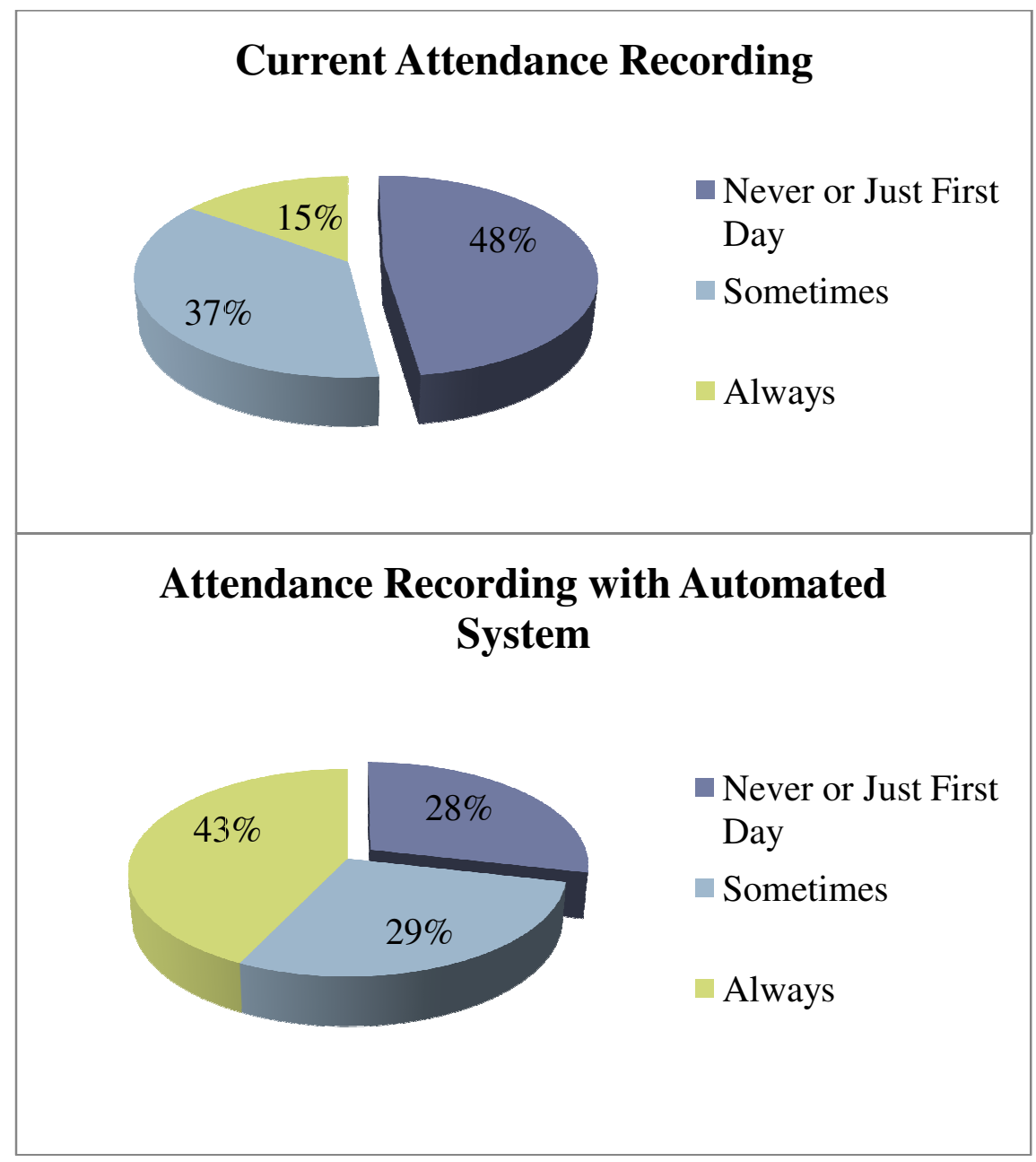

Figure 7: Survey attendance results - current system (above) v. fully automated system (below)

\section{Participation}

As mentioned previously, the inability to recognize a student leads to other classroom management problems, including in-class participation grading. In-class participation, in this sense, is defined as speaking up in class during discussions, or adding insightful comments, but not simply attending class. If a professor cannot recognize every student in the class, then it becomes very difficult to accurately and fairly record participation. Professors at Cal Poly were asked to describe their methods of recording participation, and the results showed that $24 \%$ made real-time notes as the 
student was speaking, $14 \%$ made notes after class on who they remembered participating, and 31\% based the final students' participation grades on a gist or feel for who had been participating during the 10 week grading period. To accurately grade students using each of these methods (a total of $66 \%$ of the methods professors use) requires the ability to recognize and name every student in the class; a professor cannot mark down a student for participating if the student's identity is unknown. In addition, the latter two methods require professors to have a perfect memory of which students participated as far back as an entire quarter (10 weeks). Consequently, these factors lead to a high probability of grading error, and a need for a better quantitative system to record participation.

To design a better system to record participation, the deficiencies of the current system must be known. The professors that do not record participation in all of their classes were asked to give their reasons for doing so. Because the professors were not limited to a single reason, Figure 8 shows the amount of responses for each reason, and not the percentages. The top two reasons given where that participation grading is too time consuming, and that there are too many students. Thus, an ideal system should be extremely quick, and able to handle a large amount of students. 


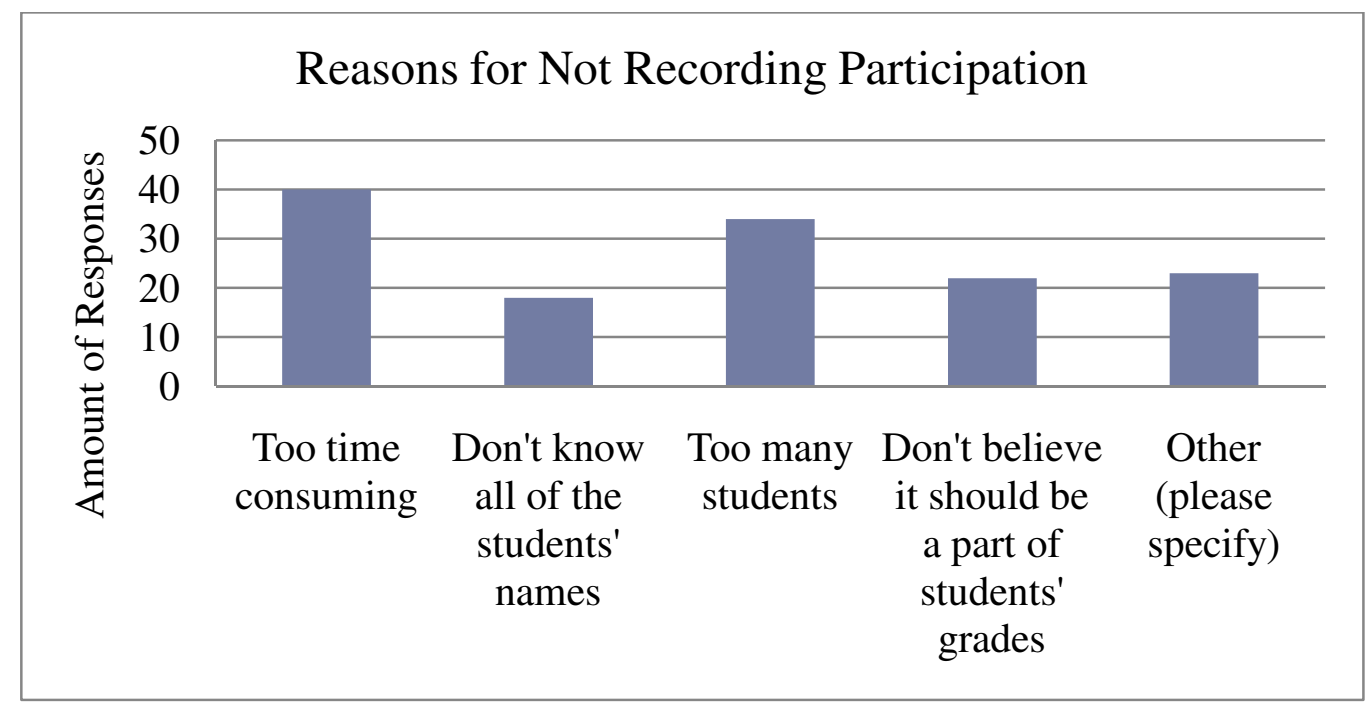

Figure 8: Survey participation results - reasons for not recording participation

To assess professors' desire for a better participation recording method, the survey included a question asking in how many classes is participation recorded in some form, and a second question asking in how many classes would participation be recorded with an easier method. This easier method is defined as an "easy way to identify a student and record his or her participation in a few seconds even in large classes." The results, illustrated in Figure 9, show a $45 \%$ reduction (from $40 \%-22 \%$ ) in professors not grading participation in any class with the easier system. This provides the evidence of the need for a better recording method. 


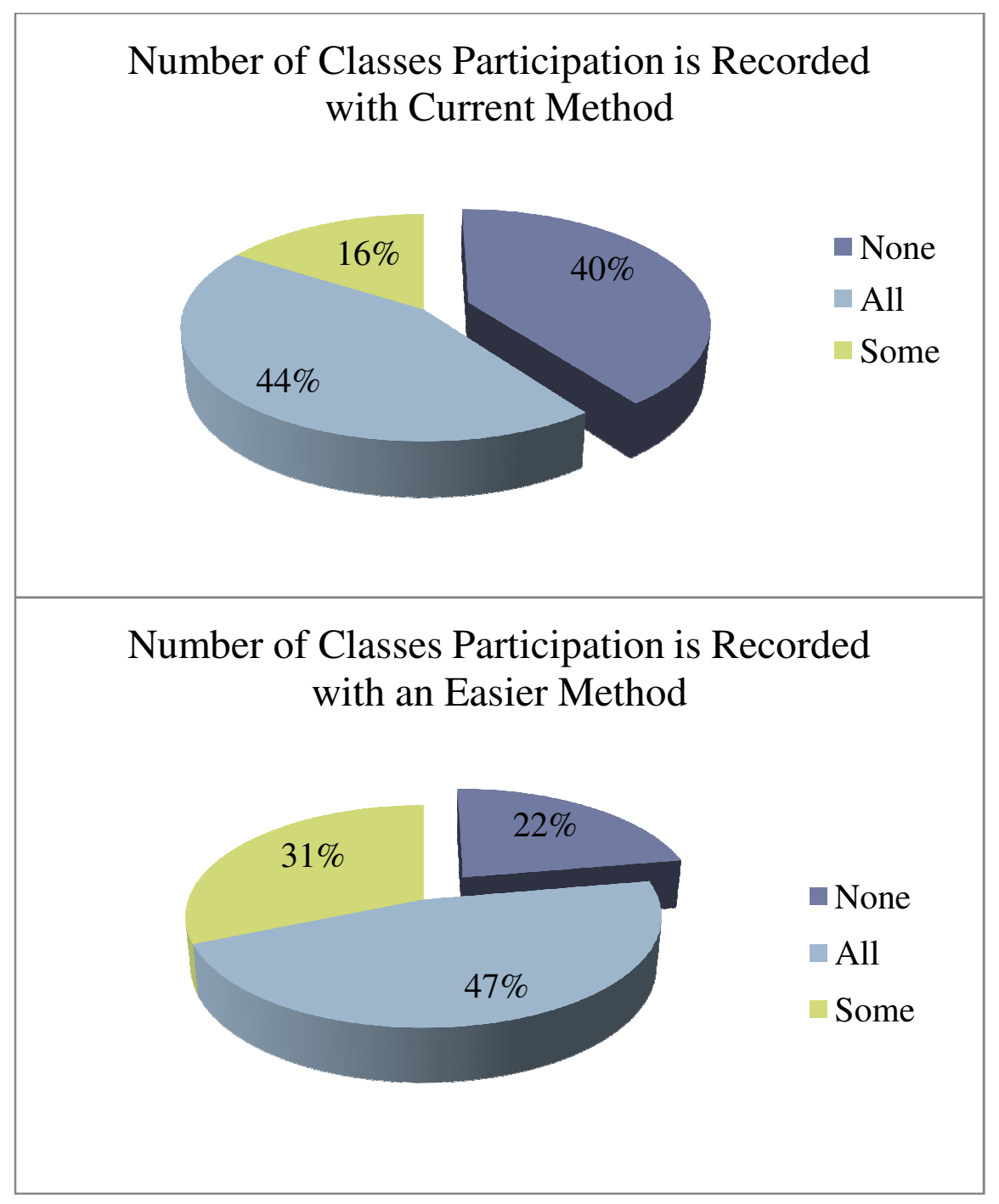

Figure 9: Survey participation results - current system (above) v. easier system (below)

\section{System Design}

The analysis of the survey results established a need for a system which improves attendance and participation recording. Furthermore, a system which provides a professor with a way to learn students' names can only help with participation and attendance recording. Specifically, an ideal system will have the following characteristics: 
- Automatically record attendance

- Quickly assist a professor in recording participation in real time

- Save information in a accessible manner

- Provide learning aid for recognizing and identifying students

A system which accomplishes these goals will increase both classroom performance, and fair and accurate participation grading, and decrease the time wasted in class. It also has the potential to create a more personalized student-teacher relationship.

\section{Formulation of RFID System}

The next generation of Cal Poly's student identification (ID) cards will contain an RFID tag. While the tag will be passive HF, having a read distance of only a few centimeters, it is assumed by the university's new interest in RFID that any type of passive tag can be placed inside the ID card. This provides a unique opportunity to utilize RFID technology as a classroom management tool.

As previously discussed, existing RFID classroom systems can locate students to a particular classroom, and take attendance in doing so, but they offer no solutions to aid professors in learning students' names or recording in-class participation. By locating students within a classroom, an RFID system has the potential to solve both of these deficiencies. This can be achieved by presenting the information of students' locations to the professor in an understandable and intuitive manner, and providing a system to assist in both participation recording, and student recognition. 


\section{User Interface}

To meet these design requirements, an interactive user interface on a mobile device is necessary. Almost half of all surveyed professors preferred a tablet device over a laptop or smart phone. Therefore, in the design of the system, the professor will be equipped with a portable tablet computer, for a mobile, lightweight tool to track participation and to aid in recognizing students. The user interface will allow professors to visually see each student in the room by locating every student's picture and name in a model of the classroom. In the small-scale prototype screenshot, shown in Figure 10, the classroom is broken up into a grid of three zones, which help distinguish the relative locations of every student. Using the touchscreen device, the instructor can quickly select a student and add a participation point while the student is talking in class. Along with participation, the database of the program can record attendance for grading purposes. The user interface is a prototype example that has been created using Microsoft Access 2007 (see Appendix C: Program Code), and would require additional work for full implementation purposes.

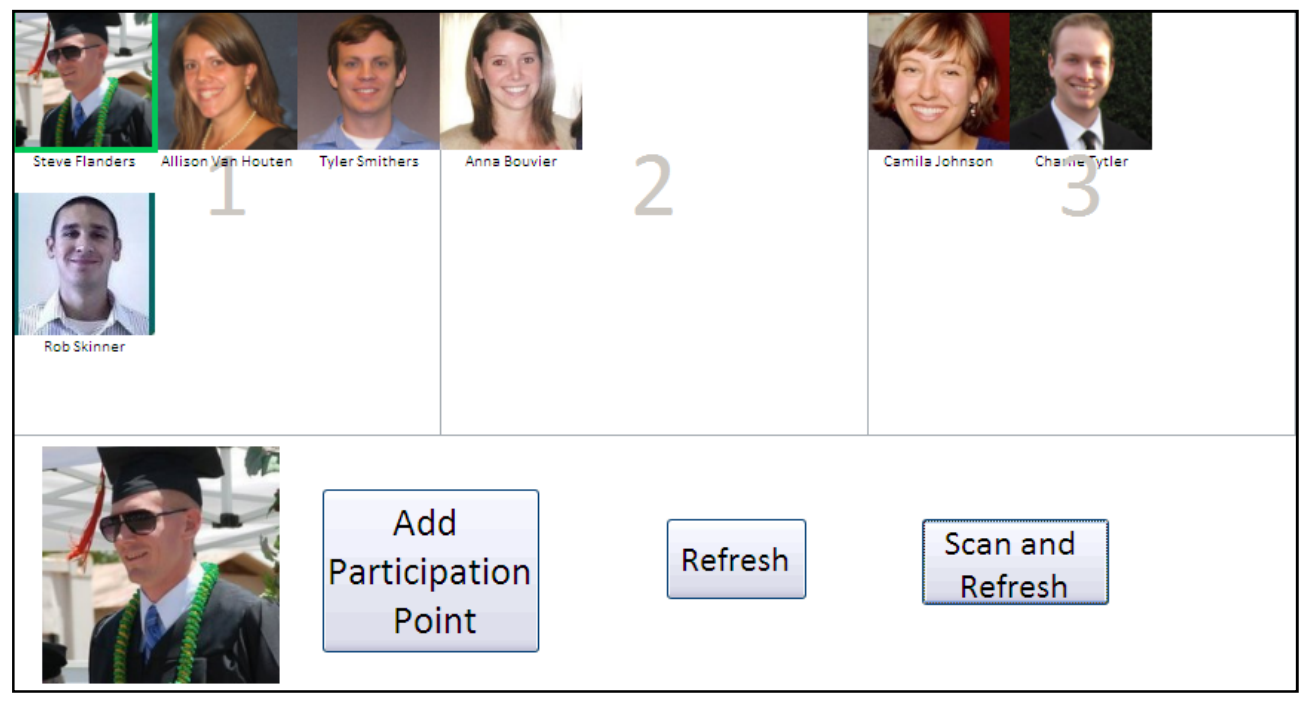

Figure 10: Screenshot of prototype user interface 
The backbone of this system is its ability to locate students relatively accurately within a classroom. Developing a localization method for the classroom environment is the focus of the following sections.

\section{Localization}

Before developing the localization system, the type of RFID system to be used, and the type of localization method must both be evaluated.

When considering inserting tags into twenty or thirty thousand student identification cards, passive tags become a much more cost effective option. This fact, coupled with read ranges of up to 10 meters, make the UHF passive RFID system the ideal choice for a classroom management system. However, when compared to HF systems, the cost per UHF reader is an order of magnitude greater than the cost of an HF reader, with prices generally ranging from $\$ 800$ - $\$ 1600$. Even though a UHF reader can connect to four antennas, each with read ranges of up to 10 meters, multiple localized HF readers are a cost effective option to consider.

The distance estimation method of localization is very accurate, but requires many custom algorithms and metrics that are not readily available in commercial systems. The simplicity of the proximity approach makes it appealing, as well as its versatility to indoor environments. While accuracy is important, locating a student in a large classroom with the help of a visual display does not require the level of accuracy that the distance estimation method provides. The goal of the system is to provide the teacher with a general vicinity of a student. In addition, the proximity method's accuracy is 
dependent on the density and number of antennae, making it a very customizable option. For these reasons, the optimal localization method is the proximity approach.

\section{Localization System Designs}

There are several designs of the localization techniques that are tested and evaluated in a classroom setting to insure the accuracy of the system. Three separate system configurations are presented in the following sections and evaluated in Chapter IV.

\section{Ceiling Corner Four-Antenna System}

Because RFID systems generally get much more expensive as their complexity increases, the first design involves only one reader and four antennas and will test if a highly simplistic design is effective enough to be a viable classroom management tool. The basic structure of this configuration places each antenna at the highest corners of a room where the walls meet the ceiling, and positions them so they are all facing directly to the middle of the room. The proximity localization method is the basis behind this design. Figure 11 illustrates the system configuration, and shows four antennas, represented as different colored squares in the corners of the room. The colored half circles represent the range of each antenna. These four antenna fields and their overlap create nine separate zones. In reality, because antenna ranges are not perfect spheres, this depiction is not as exact as shown, but it does show the basic idea behind the design. By creating these nine different zones, a tag's location can be determined to be within a small area. For instance, if a tag is read by both Antenna 1 and Antenna 2, then the tag 
should be located within zone 2 . Zone 9 is unique because it actually contains five different overlap zones, but due to their small size, they are combined to form one central zone. As a result, if a tag is read by any three antennas, or all four, the tag should be located in the middle of the room where zone 9 is.

In theory, this method should locate tags accurately, but there are many factors that can interfere with the transmissions of an antenna. As discussed previously, the absorption of radio waves by the water in human bodies and wave reflection off of metal objects are two main interferences to consider.

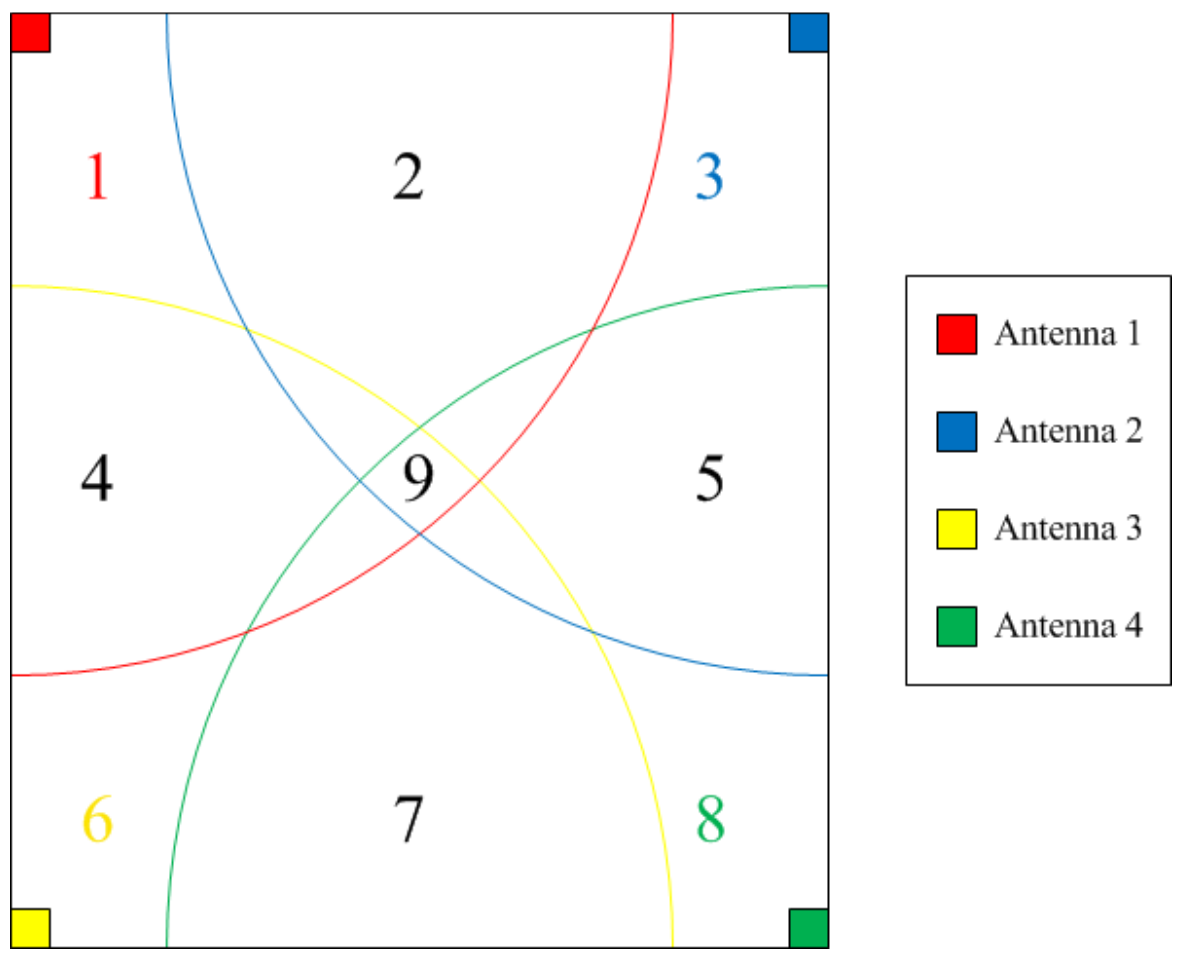

Figure 11: Proximity Ceiling Corner Four-Antenna System 


\section{Overhead Multiple-Antenna System}

The ceiling corner configuration has several design flaws exposed later through experimentation. Thus, the overhead configuration is a secondary refinement, designed to minimize the deficiencies of the previous system. While the overhead configuration is similar to the ceiling corner system, in that it uses the proximity localization method, distinct changes are evident between the two systems. First, the overhead model is much more customizable because it is not limited to the four corners of a room. Although Figure 12 shows nine separate zones and four antennas, the system can be configured with as many antennas that are required to cover the area of a classroom. Second, because the antennas are directed towards the floor, which is typically a distance no more than four meters, the required read distance is much less than the distance from a ceiling corner. Lastly, the absorption by students sitting in the classroom, and the resultant interference with tag readability, should be reduced by the overhead configuration. If students place their ID cards on the desks they are sitting at to sign in to the system, the overhead antenna will have must better line of sight to the tag, which is necessary in the presence of water. Conversely, a ceiling corner configuration does not have nearly as clear of a path to the tags. Therefore, the overhead design configuration should lead to more accurate read rates. 


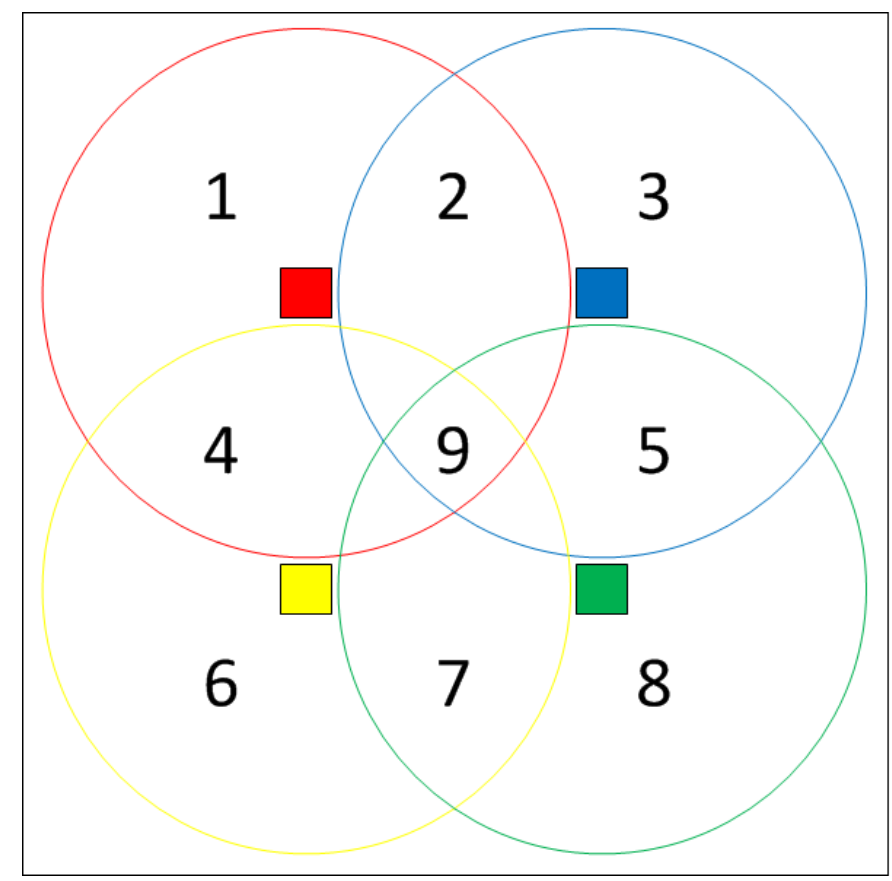

\begin{tabular}{|c|}
\hline Antenna 1 \\
\hline Antenna 2 \\
\hline Antenna 3 \\
\hline Antenna 4 \\
\hline
\end{tabular}

Figure 12: Proximity Overhead Multiple-Antenna Design

\section{Local HF Reader System}

This design uses several small RFID readers located at every large table or cluster of desks in a classroom. HF readers are much smaller than UHF readers, about the size of a computer mouse, and would not take up significant room. These HF readers require students to pass their identification card within a three inch proximity of the reader at the beginning of each class. The largest benefit of this system is that it guarantees the location accuracy of every tag in the classroom. A severe drawback to the system is that it requires a great deal of infrastructure and money to implement because of the work required wiring so many readers. Due to this, the system will not be a viable option for most classrooms, although it will be ideal in many new business classrooms that have complex, preexisting electrical infrastructure at every table. 


\section{Methodology}

Experimentation and testing is necessary to evaluate the accuracy and characteristics of each proposed system. The results from the experiments provide vital information that can be used to perfect the configuration of each system as well as choose the optimal design for a given condition. Also, it is assumed that the local HF reader configuration does not require testing, and will produce near $100 \%$ read accuracy. This can be justified by including a visual control into the design, such as a small light, to show the student whether his or her card has been read. Therefore, this chapter discusses the testing of the two passive UHF systems, for which accuracies and read ranges are unknown.

\section{Ceiling Corner Four-Antenna System}

The goal of the experimentation is to establish a probability distribution for the read rate (or accuracy) of the system. By knowing the probability distribution, the system can be further customized to more accurately interpret the data of a given scan.

\section{Experimental Design}

The four antennas are mounted in each corner of a 12' x 16' room and positioned to face directly to the center of the room. The room is divided into a grid of thirty-two rectangular sections (Figure 13), each measuring 2' x 3'. The section dimensions are chosen as such, because they reproduce the average space that one student occupies inside a classroom. After careful measurements, tape is laid down on the floor of the 
room along the lines of the grid to provide a visual method of determining the location of each section. To suspend the RFID tags, string is strung across the room at 38" above the ground. If the grid of the room (Figure 13, left) is regarded as four columns and eight rows, there are eight pieces of string running across the entire length of the eight rows. Eight, simple PVC structures, seen in Figure 14, are built to support the string, and are placed down the middle of the room, between the second and third columns (Figure 13, right). The string is threaded through two drilled holes in the PVC, at 38 " above the ground. Above the middle of each of these sections, an RFID tag is taped to a two inch piece of plastic straw and attached to the string. The strings and tags are measured and adjusted so that they are suspended exactly three feet above the floor.

\begin{tabular}{|c|c|c|c|}
\hline 1 & 2 & 3 & 4 \\
\hline 5 & 6 & 7 & 8 \\
\hline 9 & 10 & 11 & 12 \\
\hline 13 & 14 & 15 & 16 \\
\hline 17 & 18 & 19 & 20 \\
\hline 21 & 22 & 23 & 24 \\
\hline 25 & 26 & 27 & 28 \\
\hline 29 & 30 & 31 & 32 \\
\hline
\end{tabular}
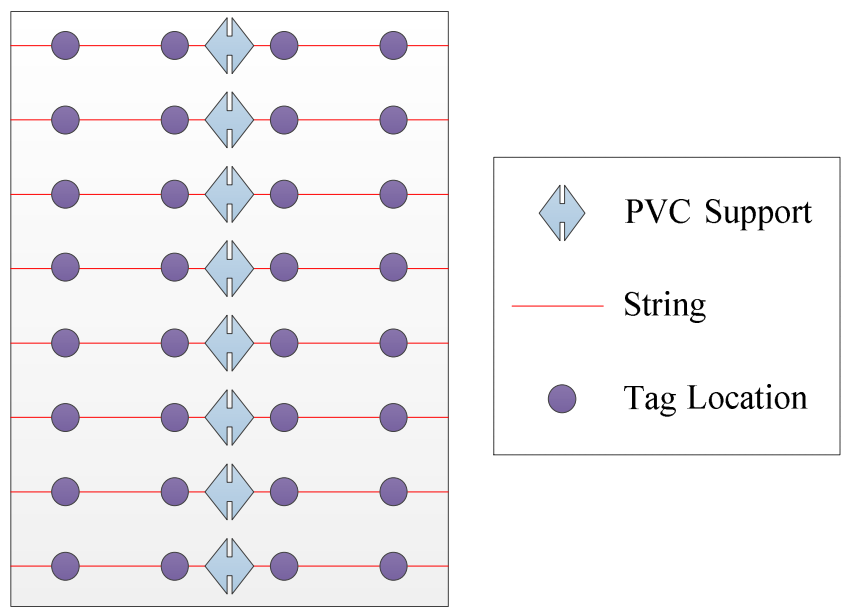

Figure 13: 12' x 16' experiment room grid (left); PVC, string, and RFID tag positions (right)

\section{Experimental Procedure}

For the purposes of reducing the experimentation time, each column, consisting of eight suspended tags, is tested individually. The RFID reader is turned on to detect tags 
for 10 seconds, and then is turned off. Data is recorded that shows which tags are read by which antennas. The tags are left in the same positions, and the process is repeated two more times. After the third replication, the current column is exhausted, and the tags are moved to a different column. After the four different columns have been tested, a sequence is finished. The column order of the eight tags is randomly recalculated, and another sequence begins. In all, the experiment lasts three sequences, resulting in thirtysix different replications, and almost 1000 data points.

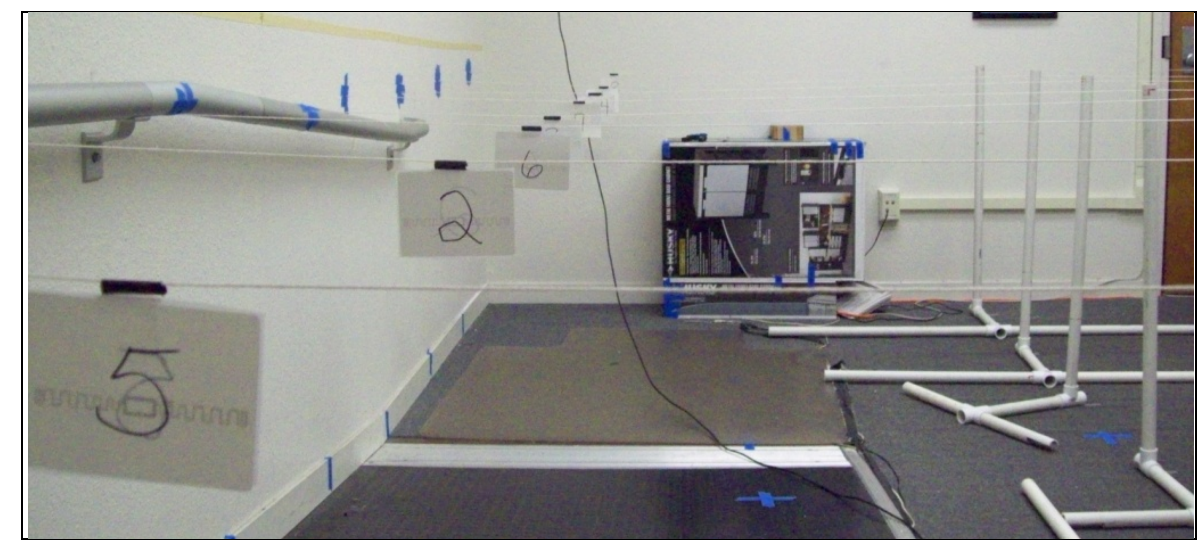

Figure 14: Setup of Control Room with Suspended Tags

\section{Overhead Multiple-Antenna System}

The results of the first experiment, as discussed in more detail late in the Results chapter, exposed the design flaws of not only the system configuration, but the experimentation itself. Consequently, the experimental design and procedure were completely redesigned for the overhead multiple-antenna system testing. 


\section{Experimental Design}

Although this experiment has the same goals as the previous experiment - to determine a probability distribution of the accuracy of the system - it differs much in its approach. This method assumes that if a probability distribution of the accuracy of one antenna is determined, the same statics can be applied to every antenna in the system. Furthermore, the number of antennas required in this configuration is unknown before the read range and accuracy of one antenna is determined. Therefore, it is unnecessary to test multiple antennas at once as the previous experiment did.

Additionally, the tag position in this experiment will be changed to better represent an implemented system. Instead of hanging vertically from strings, the tags are placed on the top of a desk, to provide more realistic results. To do this, tags are adhered to four pieces of cardboard, each measuring 3' $x$ 4', and the cardboard is placed flat onto the top of the desk. The cardboard pieces are divided into a grid of 1' x 1' cells, and four tags are placed in every cell, which altogether total 192 tags. The antenna is installed in the ceiling by replacing a panel with a custom made piece of plywood with the same dimensions. The antenna is simple screwed into the plywood, and positioned in the ceiling. The distance from the antenna to the top of the desks below is $7.42 \mathrm{ft}$ ( $89 \mathrm{in}$.).

The experiment will test three main factors: tag orientation, read duration, and scan method, each shown in Table 3. To test the tag orientation factor, the four tags placed in each 1' x 1' cell will be arranged in the manner illustrated in Figure 15 (left). 
Table 3: Factors and levels of experiment

\begin{tabular}{|l|l|}
\hline Factor & Levels \\
\hline Tag Orientation & $0^{\circ}, 45^{\circ}, 90^{\circ}, 135^{\circ}$ \\
\hline Read Duration (sec) & $80,120,300$ \\
\hline Scan Method & Stationary, Antenna Rotation, Tag Rotation \\
\hline
\end{tabular}

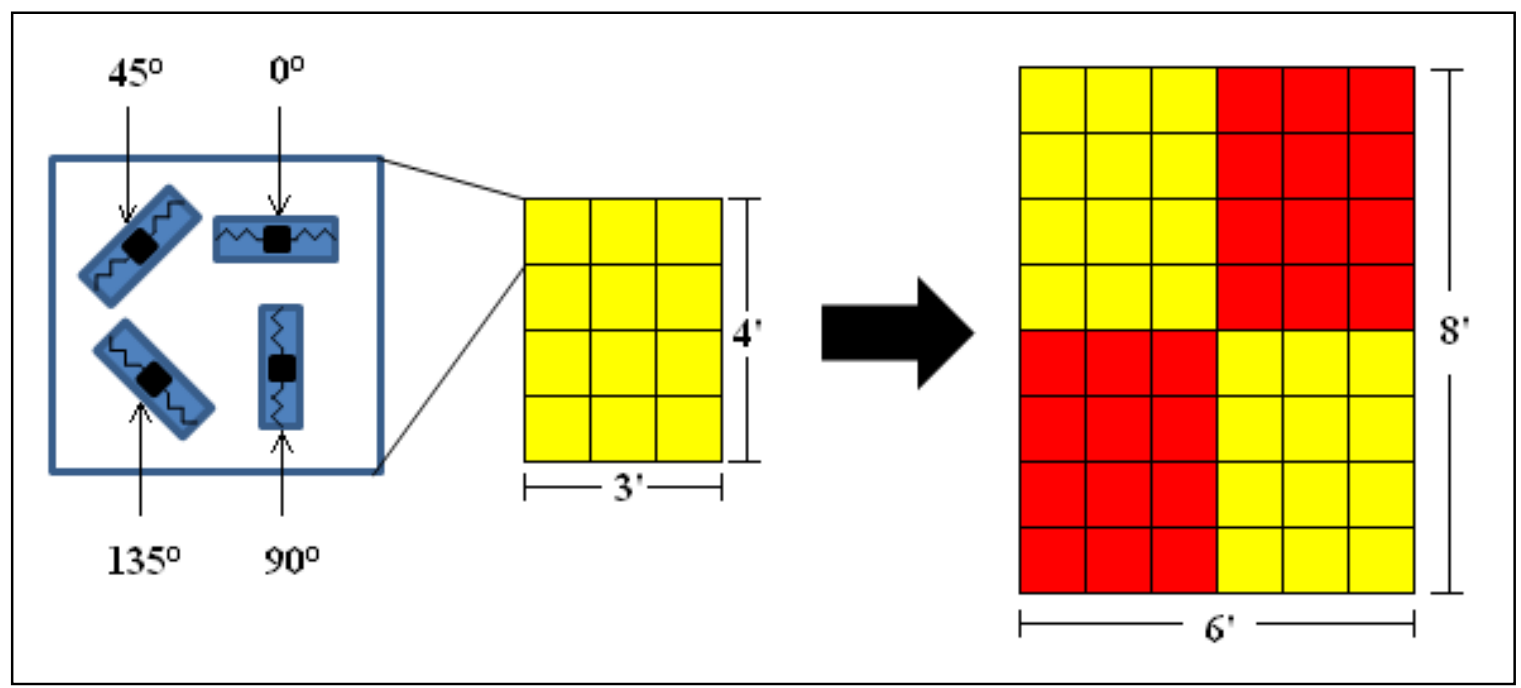

Figure 15: Tag board construction (left) and initial testing layout (right)

The experiment takes place in two phases. The first tests a 6' x 6' area directly beneath the antenna, by placing them in the configuration shown in Figure 16. Because of the dimensions of the four cardboard pieces, the area actually measures 6' x 8', but only the 6' x 6' data is analyzed. From the analysis of the first phase, an optimal method is selected. This method is further tested in the second phase of experimentation which expands the initial 6' x 6' area to the edges of the read range. 


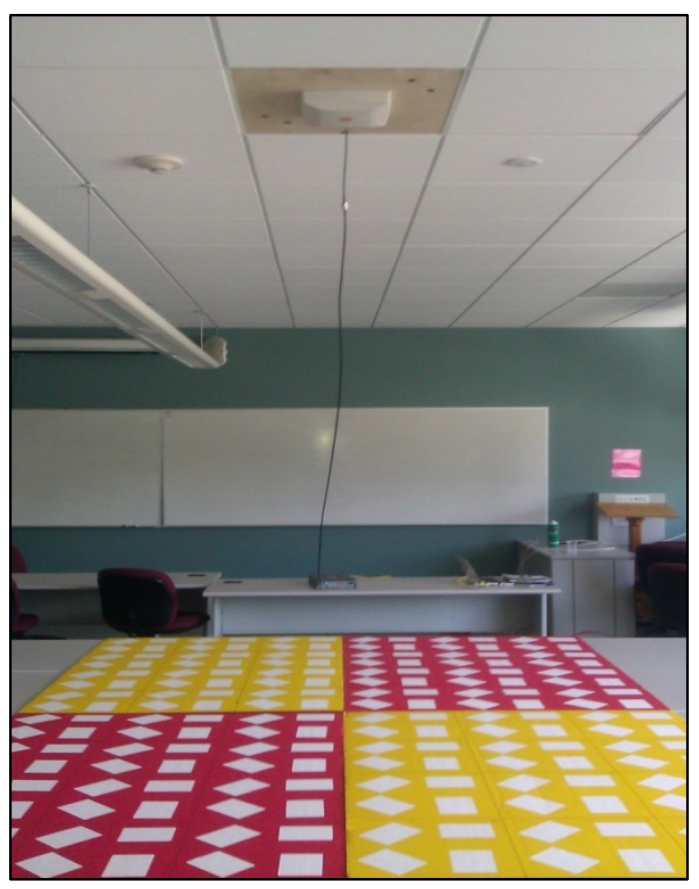

Figure 16: Initial 6’ x 8' tag board orientation

\section{Experimental Procedure}

For the initial testing, the 6' x 6' area is centered directly beneath the antenna. For every test, four repetitions are performed by rotating the four cardboard pieces one space clockwise. The read rates of every tag are recorded, and because the tags were placed on the boards in a specific order, the orientation and location of every tag is already known. To rotate the tags, the cardboard pieces are taped together and manually rotated 360 degrees about the center evenly over the course of the read duration.

Rotating the antenna is achieved by installing a Lazy Susan between the antenna and plywood board, and then manually rotating it evenly over the course of the read duration.

Table 4: Initial experimentation

\begin{tabular}{|l|l|r|}
\hline Test Name & Scan Method & Read Duration (sec) \\
\hline $80 \mathrm{~s}$ & Stationary & 80 \\
\hline $120 \mathrm{~s}$ & Stationary & 120 \\
\hline Ant 360 & Antenna 360 & 80 \\
\hline Tag 360 & Tag 360 & 80 \\
\hline
\end{tabular}




\section{Results}

All results may be referred to in Appendix B: Experiment Data and Results. The accuracy of a localization method is important because attendance and participation can be impacted. At this stage, to be considered a viable system, read rates above $90 \%$ are required, and those above $95 \%$ are desirable. This chapter presents and analyzes the results of the experimentation of Chapter IV.

\section{Ceiling Corner Four-Antenna System}

Because the room is symmetrical about both the horizontal and vertical medians, the data is effectively repeated four times, and therefore has been consolidated into the single repetitive piece. This piece, which is a $2 \times 4$ rectangle shown in Figure 17, gives the read accuracy of each section relative to the four different positions of the antennas. Relative to every section are four antennas that are located diagonally across, vertically adjacent, horizontally adjacent, and nearest to it. These relative antenna positions are shown as the colored boxes outside of the grid. This symmetry allows all the data for each of the antenna relationships to be separated and analyzed. This attempts to reduce confounding factors such as interference in specific places in the room, or variation in antenna performance.

Unfortunately, the results are not good. Figure 17 clearly demonstrates the sporadic and poor read rates of this system. The only clear tendencies that can be inferred about the system are that sections along the longer walls are read with the lowest accuracy, while sections in the middle of the room are read with the highest. The 
difference in these read accuracies is not clear enough to provide an adequate location system.

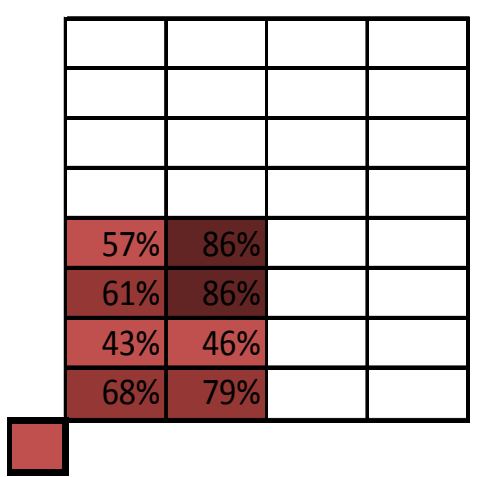

Nearest

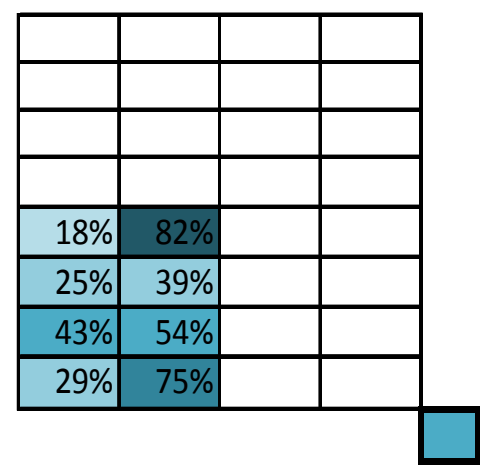

Far Horizontal

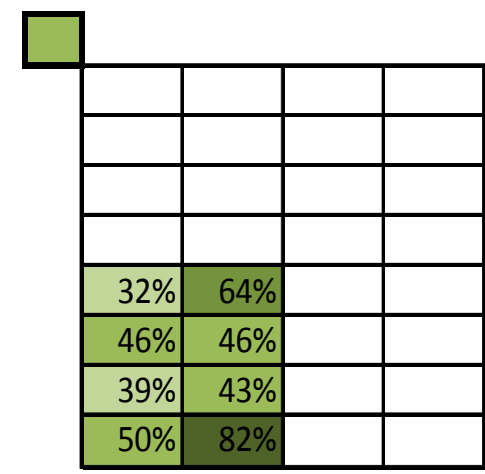

Far Vertical

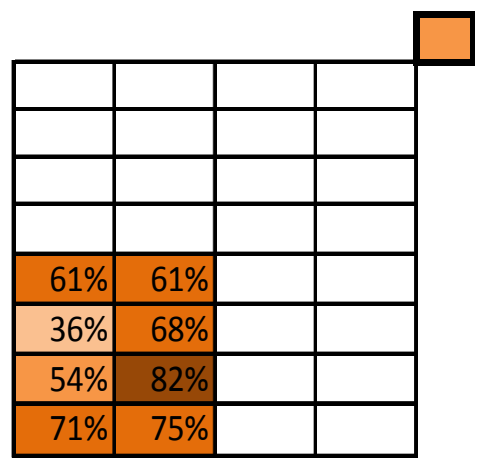

Across

Figure 17: Perspective read rates of Ceiling Corner Four-Antenna System

\section{Overhead Multiple-Antenna System}

The experimentation tested the effects of tag orientation, read duration, and scanning method, and the results of these experiments are shown in Table 5. The following statistical analyses can be found in Appendix A: Statistical Analyses of Results. The analysis of variance (ANOVA) statistical test proves that there is a significant difference between the 45 degree orientation and the other three orientations. 
The results show that the 45 degree orientation produces a greater read rate than any other orientation (see Figure 18; Appendix A: Significance of Tag Orientation). Through all four experiments it consistently produced read rates of $92 \%$ or better. Additionally, the results show that the read duration of 180 seconds produces significantly greater read rates than that of 80 second duration $(p$-value $=.002)$ (see Appendix A: Significance of Read Duration).

Lastly, the three scanning methods (stationary, antenna rotation, and tag board rotation) produced read rates that are significantly different from each other, proving that scan method affects the readability of a tag (see Appendix A: Significance of Scan Method). Rotating the group of tag boards about the middle produced a 100\% read rate. This proves that moving a tag through the field of the antenna has a significant effect on the readability of that tag. In addition, rotating the antenna did not produce the same level of results as rotating the tags did, but it did show an improvement in the read rate over the stationary method. While the act of rotating around in a circle about the center of the antenna is not the most applicable procedure to a classroom, the important thing to conclude is that any sort of movement with the tag will result in an increase in readability. 


\begin{tabular}{|l|l|l|l|l|l|}
\hline $25 \%$ & $31 \%$ & $50 \%$ & $50 \%$ & $75 \%$ & $100 \%$ \\
\hline $63 \%$ & $63 \%$ & $25 \%$ & $50 \%$ & $94 \%$ & $100 \%$ \\
\hline $50 \%$ & $100 \%$ & $50 \%$ & $50 \%$ & $94 \%$ & $100 \%$ \\
\hline $38 \%$ & $100 \%$ & $25 \%$ & $38 \%$ & $63 \%$ & $100 \%$ \\
\hline $88 \%$ & $63 \%$ & $50 \%$ & $38 \%$ & $25 \%$ & $94 \%$ \\
\hline $75 \%$ & $100 \%$ & $44 \%$ & $44 \%$ & $63 \%$ & $100 \%$ \\
\hline $100 \%$ & $50 \%$ & $94 \%$ & $31 \%$ & $44 \%$ & $94 \%$ \\
\hline $25 \%$ & $25 \%$ & $75 \%$ & $100 \%$ & $94 \%$ & $50 \%$ \\
\hline
\end{tabular}

\begin{tabular}{|l|l|l|l|l|l|}
\hline $50 \%$ & $100 \%$ & $100 \%$ & $88 \%$ & $100 \%$ & $25 \%$ \\
\hline $100 \%$ & $100 \%$ & $100 \%$ & $100 \%$ & $100 \%$ & $100 \%$ \\
\hline $56 \%$ & $100 \%$ & $100 \%$ & $100 \%$ & $100 \%$ & $100 \%$ \\
\hline $88 \%$ & $100 \%$ & $100 \%$ & $100 \%$ & $100 \%$ & $100 \%$ \\
\hline $100 \%$ & $100 \%$ & $100 \%$ & $100 \%$ & $100 \%$ & $88 \%$ \\
\hline $25 \%$ & $100 \%$ & $100 \%$ & $100 \%$ & $100 \%$ & $100 \%$ \\
\hline $100 \%$ & $100 \%$ & $100 \%$ & $100 \%$ & $100 \%$ & $88 \%$ \\
\hline $25 \%$ & $38 \%$ & $100 \%$ & $100 \%$ & $100 \%$ & $100 \%$ \\
\hline
\end{tabular}

\begin{tabular}{|l|l|l|l|l|l|}
\hline $31 \%$ & $94 \%$ & $81 \%$ & $56 \%$ & $100 \%$ & $100 \%$ \\
\hline $25 \%$ & $100 \%$ & $25 \%$ & $69 \%$ & $100 \%$ & $88 \%$ \\
\hline $44 \%$ & $44 \%$ & $81 \%$ & $94 \%$ & $44 \%$ & $69 \%$ \\
\hline $25 \%$ & $100 \%$ & $100 \%$ & $94 \%$ & $100 \%$ & $100 \%$ \\
\hline $100 \%$ & $50 \%$ & $100 \%$ & $100 \%$ & $100 \%$ & $100 \%$ \\
\hline $25 \%$ & $100 \%$ & $88 \%$ & $100 \%$ & $100 \%$ & $100 \%$ \\
\hline $100 \%$ & $31 \%$ & $56 \%$ & $81 \%$ & $63 \%$ & $81 \%$ \\
\hline $25 \%$ & $50 \%$ & $44 \%$ & $31 \%$ & $94 \%$ & $25 \%$ \\
\hline
\end{tabular}

\begin{tabular}{|l|l|l|l|l|l|}
\hline \multicolumn{5}{|c|}{135} & \\
\hline $100 \%$ & $25 \%$ & $75 \%$ & $38 \%$ & $25 \%$ & $50 \%$ \\
\hline $75 \%$ & $25 \%$ & $100 \%$ & $100 \%$ & $100 \%$ & $100 \%$ \\
\hline $100 \%$ & $38 \%$ & $100 \%$ & $100 \%$ & $100 \%$ & $100 \%$ \\
\hline $100 \%$ & $63 \%$ & $100 \%$ & $100 \%$ & $100 \%$ & $100 \%$ \\
\hline $100 \%$ & $69 \%$ & $100 \%$ & $100 \%$ & $100 \%$ & $100 \%$ \\
\hline $100 \%$ & $25 \%$ & $100 \%$ & $100 \%$ & $100 \%$ & $100 \%$ \\
\hline $100 \%$ & $25 \%$ & $81 \%$ & $69 \%$ & $75 \%$ & $31 \%$ \\
\hline $69 \%$ & $31 \%$ & $50 \%$ & $50 \%$ & $31 \%$ & $31 \%$ \\
\hline
\end{tabular}

Figure 18: Tag orientation read rate results

Table 5: 6' x 6' Read rates of multiple experiments

\begin{tabular}{|l|r|r|r|r|}
\hline & \multicolumn{1}{|c|}{$80 \mathrm{sec}$} & $180 \mathrm{sec}$ & Ant 360 & Tag 360 \\
\hline Average & $69 \%$ & $73 \%$ & $83 \%$ & $100 \%$ \\
\hline 135 & $81 \%$ & $79 \%$ & $82 \%$ & $100 \%$ \\
\hline 090 & $65 \%$ & $69 \%$ & $74 \%$ & $100 \%$ \\
\hline 045 & $92 \%$ & $94 \%$ & $97 \%$ & $100 \%$ \\
\hline 000 & $38 \%$ & $49 \%$ & $79 \%$ & $100 \%$ \\
\hline
\end{tabular}

The results from the preliminary experimentation show that the best discovered method is to rotate the tags around the center of the antenna. However, because this is not applicable to a classroom system, it is not pursued as viable method. Similarly, 
rotating an antenna as it scans would require a great deal of knowledge and ingenuity to construct, and is outside the scope of this research. Therefore, orienting the tag at a 45 degree angle, using a stationary scan method, and a read duration of 180 seconds is the most viable and applicable method to a classroom management system, and requires additional experimentation to determine the full read range.

The second experiment expands the initial 6' x 8' area by position the tag boards farther out, as illustrated in Figure 19. The results, along with the initial testing results are shown in Figure 20.

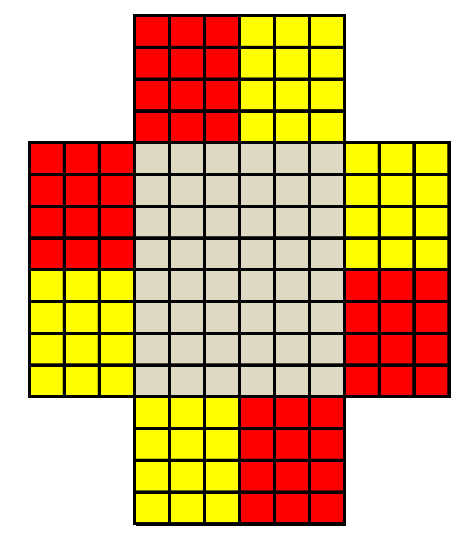

Figure 19: Positioning of tag boards in secondary testing 


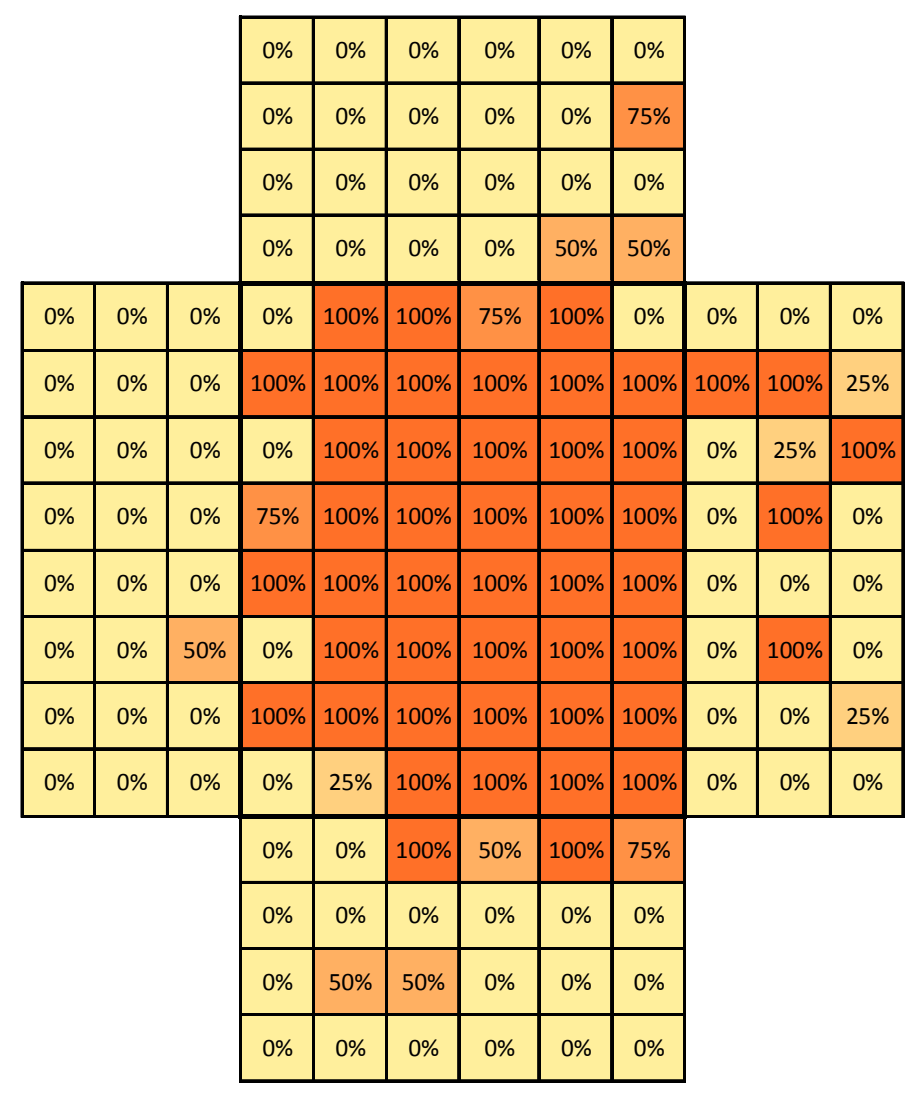

Figure 20: Extended read range results

Although worthwhile, the secondary experimentation proves that the full read range is nearly reached within the 6' x 6' area. With this data, it is now possible to design a custom system for any given classroom. An example of the expected probability plot of a two antenna system, six feet apart from each other, is given in Figure 21. 


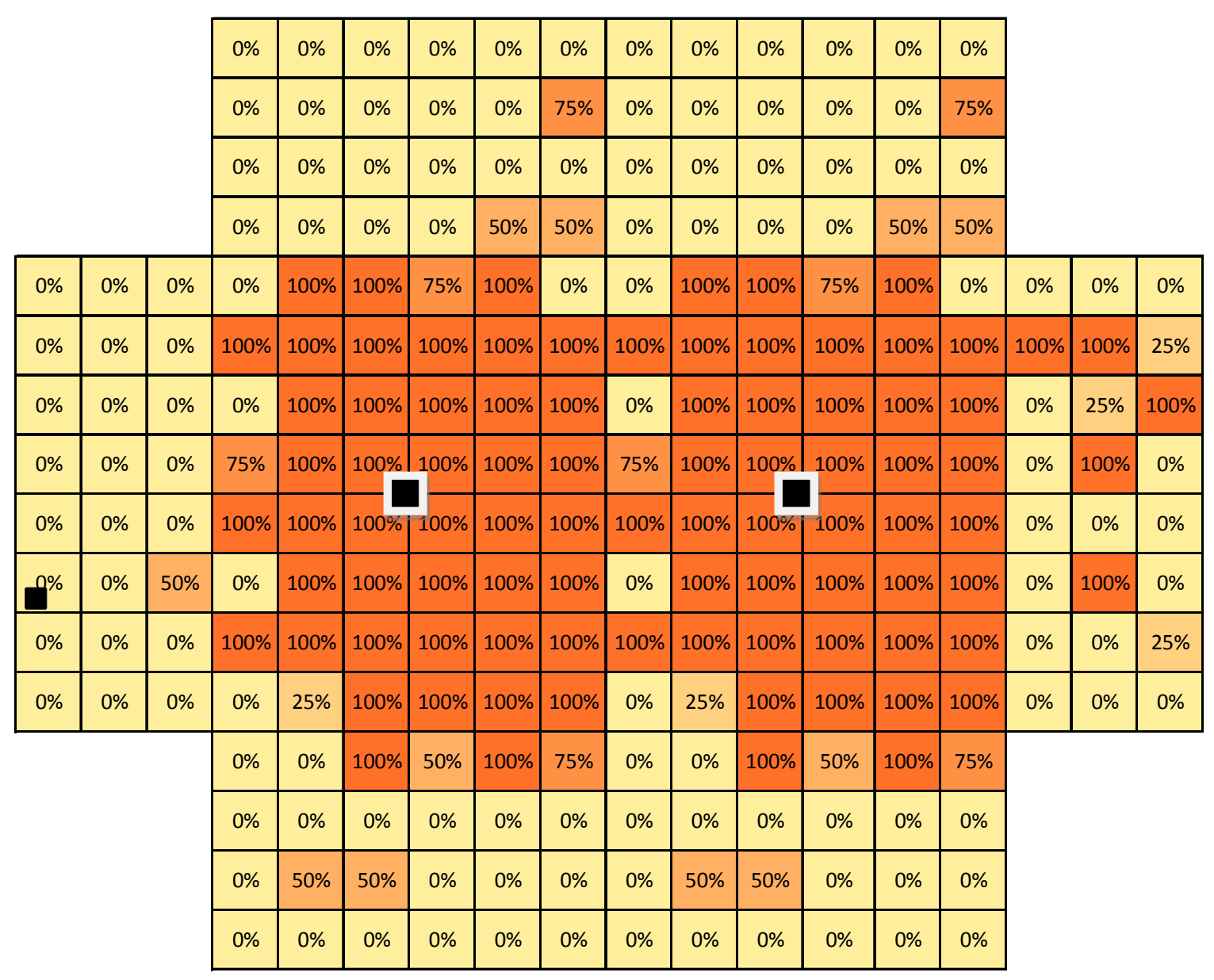

Figure 21: Two antenna system probability distribution

\section{Interfering Factors}

Because the testing of the overhead multi-antenna system is performed in an actual classroom, the environment was not completely ideal. Light fixtures, shown partially in Figure 16 hang below the ceiling near where the antenna is installed. Because the ceiling panels are 2' $\mathrm{x}$ 2', there was no way to position the antenna directly in the middle of the two light fixtures. Initially, the antenna was install three feet from the fixture, and due to poor results, was moved another panel over five feet away from the fixture. The number of tags read from each of these positions was significantly greater five feet away from the light fixture than three feet away ( $\mathrm{p}$-value $=0.054)$. The 
comparison of the tests at these two positions, shown in Table 6, provides evidence that the light fixture did indeed interfere with the readability of the tags.

In every experiment presented in this research, the antenna is two feet closer to the left light fixture than the right, which has a strong possibility to interfere with results. However, the effects of light fixtures and other interfering objects are important to understand in the design of an RFID system for a classroom.

Table 6: Light fixture interference

\begin{tabular}{|l|r|r|}
\hline & Light $80 \mathrm{sec}$ & $80 \mathrm{sec}$ \\
\hline Average & $62 \%$ & $69 \%$ \\
\hline 135 & $59 \%$ & $81 \%$ \\
\hline 090 & $69 \%$ & $65 \%$ \\
\hline 045 & $76 \%$ & $92 \%$ \\
\hline 000 & $43 \%$ & $38 \%$ \\
\hline
\end{tabular}

\section{Cost Analysis}

At first glance, the cost analysis shows that both passive UHF and HF RFID systems are expensive, with the HF system being the cheaper of the two. Additionally, the size and characteristics of the classroom can have a dramatic effect on the price of the system. The high price tag is especially difficult to justify because most of the benefits a classroom management system provides are not easy to economically value. However, automatically recording attendance does eliminate the time necessary to manually do so, therefore increasing the value of the class to the students. A very simple calculation, based on the 2010 average cost of attending a public in-state four year university of $\$ 16140$ (Baum, 2010), results in a cost per minute of lecture of $\$ .60$ per student (see Equation 1). If it is conservatively assumed that manually recording attendance takes two minutes, then the cost to take attendance is approximately $\$ 42.00$ (see Equation 2). If 
attendance is taken every class period, then the annual cost is $\$ 2520.00$ (see Equation 3).

Thus, the payback periods for the UHF and HF systems are 8.66 months and 5.25

months, respectively.

Equation 1: Student cost per minute of lecture

(\$16140/ year) $x$ (1 year/45 units) x (1 unit/1 hour/week) $x$ (1/10 weeks) $x$ (1 hour/60 min)

$=\$ .60 /$ minute

Equation 2: Cost of recording attendance

(\$.60/minute $) \times(2$ minutes $) \times(35$ students $)=\$ 42.00$

Equation 3: Annual cost of recording attendance

(\$42.00/class) x (2 classes/week) x (10 weeks/term) x (3 terms/year $)=\$ 2520.00 /$ year

Table 7: Cost per classroom of four antenna UHF RFID system

\begin{tabular}{|llrrr|}
\hline Item & Price per Unit & \multicolumn{2}{l|}{ Total Price } \\
Hardware & & & & \\
$\quad$ Motorola FX7400 RFID Reader (4 Port) & $\$$ & $1,032.00$ & $\$$ & $1,032.00$ \\
$\quad$ & $\$$ & 69.99 & $\$$ & 279.96 \\
$\quad$ Poynting Antennas (4) & $\$$ & 199.99 & $\$$ & 199.99 \\
$\quad$ Superpad 10.2" Tablet PC & $\$$ & 0.15 & $\$$ & 7.50 \\
$\quad$ Passive UHF Tags (50) & & & & \\
& & & & 300.00 \\
Installation & Total Cost & & $\$ 1,819.45$ \\
\end{tabular}

Table 8: Cost per classroom of eight reader HF RFID system

\begin{tabular}{|lcrrr|}
\hline Item & \multicolumn{2}{l|}{ Price per Unit } & \multicolumn{2}{l|}{ Total Price } \\
Hardware & & & & \\
$\quad$ HF RFID Reader (8) & $\$$ & 49.48 & $\$$ & 395.84 \\
$\quad$ Superpad 10.2" Tablet PC & $\$$ & 199.99 & $\$$ & 199.99 \\
$\quad$ HF Tags (50) & $\$$ & 0.15 & $\$$ & 7.50 \\
& & & & \\
Installation & & & $\$$ & 500.00 \\
\cline { 2 - 5 } & Total Cost & & $\$ 1,103.33$ \\
\hline
\end{tabular}


Although this analysis contains many assumptions, it is meant to point out the possibly unrealized economic benefits such a system can have. Furthermore, it does not include the benefits of accurate and fair participation grading or of a more personalized learning experience. Professors who struggle learning students' names, forget who exactly participated in class discussions, and take, or want to take, class attendance every period, will benefit the most from a classroom management system. Consequently, both RFID systems are economically viable options for professors and classrooms that would utilize the system to its potential. 


\section{Conclusions and Further Analysis}

This chapter will discuss the problems of classroom management and associated objectives of the report, along with the solution approach. A recommendation of the optimal classroom management system for particular conditions will be given, and the related benefits will be presented. Finally, further analysis will be discussed to improve current designs.

\section{Summary}

This project set out to solve the difficulties involved with managing large classes. Specifically: accurately and fairly grading in-class participation, learning students' names, and recording attendance in a timely manner. It established the need that professors have for a classroom management tool, and took advantage of new RFID trends to help develop such a device. Different RFID configurations and methods were examined and tested resulting in important factors impacting the accuracy of the system being discovered. These factors were optimized into a single applicable method to produce the best results. The system is not only cost effective, but carries along many invaluable benefits as well.

\section{System Recommendation}

The high accuracy and low cost of the localized HF reader system makes it the optimal system for most classrooms. However, the amount of infrastructure the system requires to connect and power all of the readers simply will not be viable in classrooms 
with limited energy resources. In these types of conditions, the UHF overhead multiple antenna configuration is best suited. The optimal procedure for detecting tags in this configuration is for students to "sign in" to the system at the beginning of class by placing their ID card on their desk and spinning it. While the 45 degree angle orientation was shown to have a much higher read rate than any other orientation, it was also discovered that adding motion to the system increased the read rate dramatically. This classroom management system achieves its original goals and provides professors with a tool that will:

- Increase student performance

- Aid in learning students names

- Automatically record attendance

- Provide a quantitative method to quickly and accurately record in-class participation

These improved abilities will lead to an improved student teacher relationship and personalization of the learning experience. There will be less wasted time, and an associated increase in class value. Most importantly, students will be graded fairly and accurately on their in-class participation, and will improve their performance.

\section{Further Analysis}

For full implementation of the classroom management system, additional analysis is required. Specifically, the effects of students occupying a classroom, and the subsequent signal absorption, were never tested, as all experimentation was kept under semi-ideal conditions. Additionally, although the method of spinning the ID card 
increases the probability that the tag will be read, the exact amount is unknown, and must be determined to customize a system to a particular classroom. Similarly, the effects of the motion introduced by student retrieving a card and placing it on the desk are unknown, and require further testing. 


\section{Works Cited}

Arulampalam, Wiji. Am I Missing Something? The Effects of Absence from Class on Student Performance. Tech. Coventry: University of Warwick, 2007. Print.

Baum, Sandy . "Trends in College Pricing 2010."Trends in Higher Education Series. The College Board, 2010. Print.

Bing Jiang; Fishkin, K.P.; Roy, S.; Philipose, M.; , "Unobtrusive long-range detection of passive RFID tag motion," Instrumentation and Measurement, IEEE Transactions on, vol.55, no.1, pp. 187- 196, Feb. 2006

Bouet, Matthew. RFID Tags: Positioning Principles and Localization Techniques. Laboratoire d'Informatique de Paris 6 Universit’e Pierre et Marie Curie, 2009.

"Cal Poly, San Luis Obispo." CollegeView. N.p., 2011. Web. 20 May 2011. $<$ http://www.collegeview.com/schools/cal-poly-san-luis-obispo/profiles>.

Cole, DG. (1999). Supportive classroom environments for creativity in higher education. The Journal of Creative Behavior, 33(4), 277.

Devadoss, Stephen, and John Foltz. "Evaluation of Factors Influencing Student Class Attendance and Performance." American Journal of Agricultural Economics 78.3 (1996): 499-507. Oxford University Press. Web. 10 June 2011. $<$ http://www.jstor.org/stable/1243268>.

Dobkin, Daniel M. The RF in RFID: Passive UHF RFID in Practice. Newnes, 2007. Freed, Tali, and Bryce Taylor. Optimal Layout Coverage of Passive RFID Systems. Tech. Proceedings of Industrial Engineering Research Conference, May 2008. Print.

Freed, Tali, and Mike Krist. Implementation of Passive UHF RFID at a Nuclear Power Plant Warehouse. Tech. PolyGAIT, Mar 2009. Print. 
Hekimian-Williams, Cory, Brandon Grant, Xiuwen Liu, Zhenghao Zhang, and Piyush Kumar.Accurate Localization of RFID Tags Using Phase Difference. Publication. IEEE RFID, 2010. Print.

Hightower, Jeffrey, Gaetano Borriello, and Roy Want. SpotON: An Indoor 3D Location Sensing Technology Based on RF Signal Strength. Tech. Seattle, WA: University of Washington Computer Science and Engineering, 2000. Print.

InCom Corporation, InClass. <http://www.incomcorporation.com/InClass.html> (accessed Mar 2010)

Mensch J. M., Ennis C. D. Pedagogic strategies perceived to enhance student learning in athletic training. Journal of Athletic Training. 2002;37(suppl 4):S199-S207

Moore, Randy. "Helping Students Succeed in Introductory Biology Classes: Does Improving Students’ Attendance Also Improve Their Grades?" Bioscene 29.3 (Aug. 2003): 17-25.Universiteit Stellenbosch University. Web. 10 June 2011. $<$ http://sun025.sun.ac.za>.

O'Connor, M. C. (2005). RFID Takes Attendance-and Heat. RFID Journal

O'Connor, M. C. (2010). Northern Arizona University to use existing RFID student cards for attendance tracking. RFID Journal

Sanpechuda, T., and L. Kovavisaruch. A Review of RFID Localization: Applications and Techniques. Publication. Proceedings of ECTI-CON, 2008: 769-772. Print.

Song, J.,Haas,C.H.,\&Caldas, C. H. (2007).A proximity-based method for locating RFID tagged objects. Advanced Engineering Informatics, 21, 367-376.

Taylor, Bryce. "RFID Antenna Coverage Optimization." Thesis. California Polytechnic State University, 2008. Print. 
Want, R., "An Introduction to RFID Technology," Pervasive Computing, IEEE, vol.5, no.1, pp. 25- 33, Jan.-March 2006

Wasp Barcode, WaspTime RFID Solution

<http://www.waspbarcode.com/wasptime/wasptime_rfid.asp> (accessed Mar 2010)

Weinstein, R.,"RFID: a technical overview and its application to the enterprise," IT Professional, vol.7, no.3, pp. 27- 33, May-June 2005

White, F. C. "Enhancing Class Attendance." NACTA 36 (Dec. 1992): 13-15. Print.

Zhou, Junyi, and Jing Shi. RFID Localization Algorithms and Applications-a Review. Publication. Springer Science+Business Media, LLC, 2008. IEEE Xplore. Web. 20 May 2011. <http://ieeexplore.ieee.org>. 


\section{Appendix A: Statistical Analyses of Results}

\section{Significance of Tag Orientation}

Levels of Tag Orientation

1: 0 degrees

2: 45 degrees

3: 90 degrees

4: 135 degrees

\section{One-way ANOVA: RR versus Tag Orientation}

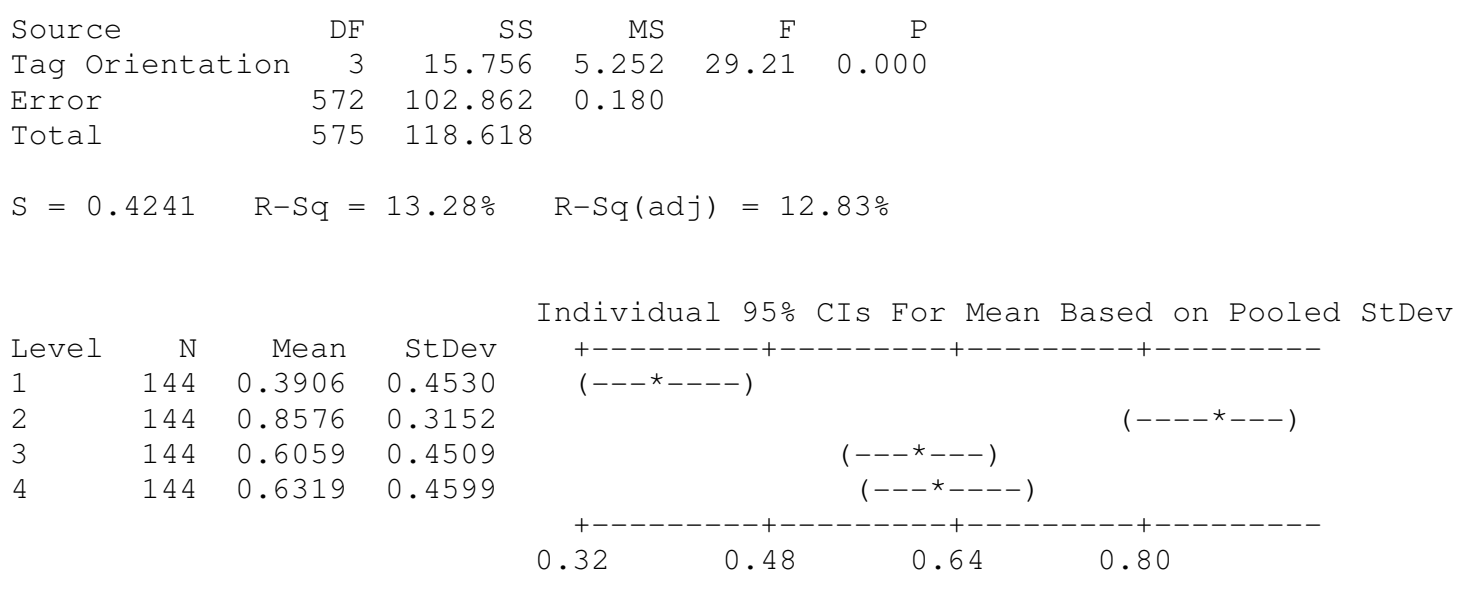

Pooled StDev $=0.4241$

\section{Grouping Information Using Tukey Method}

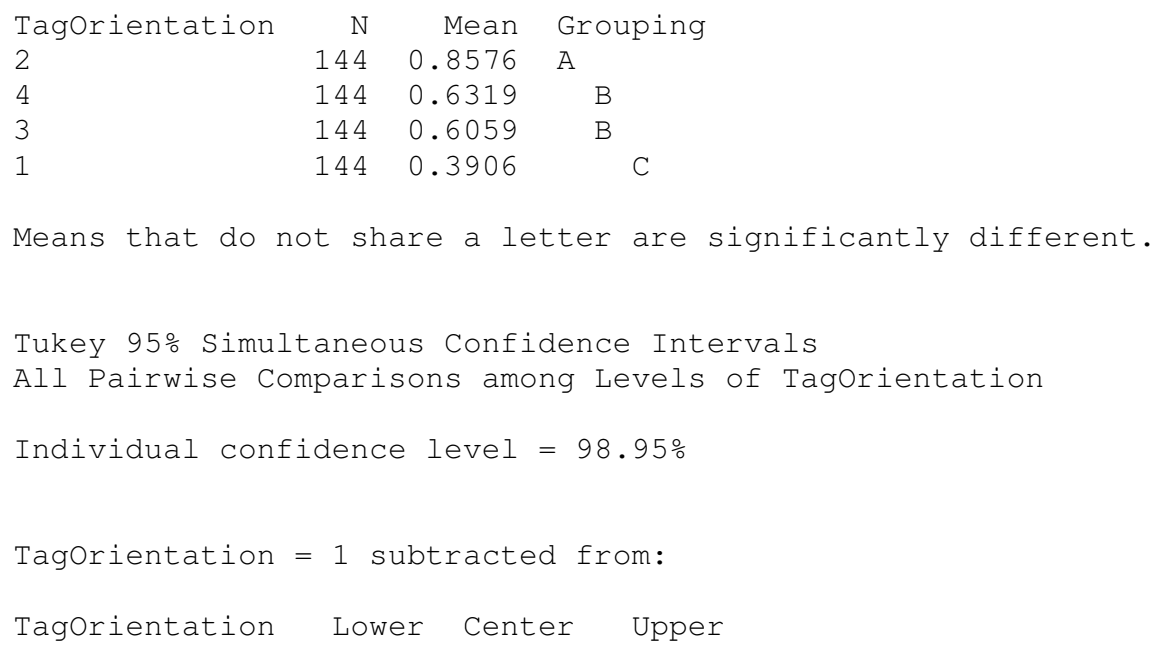




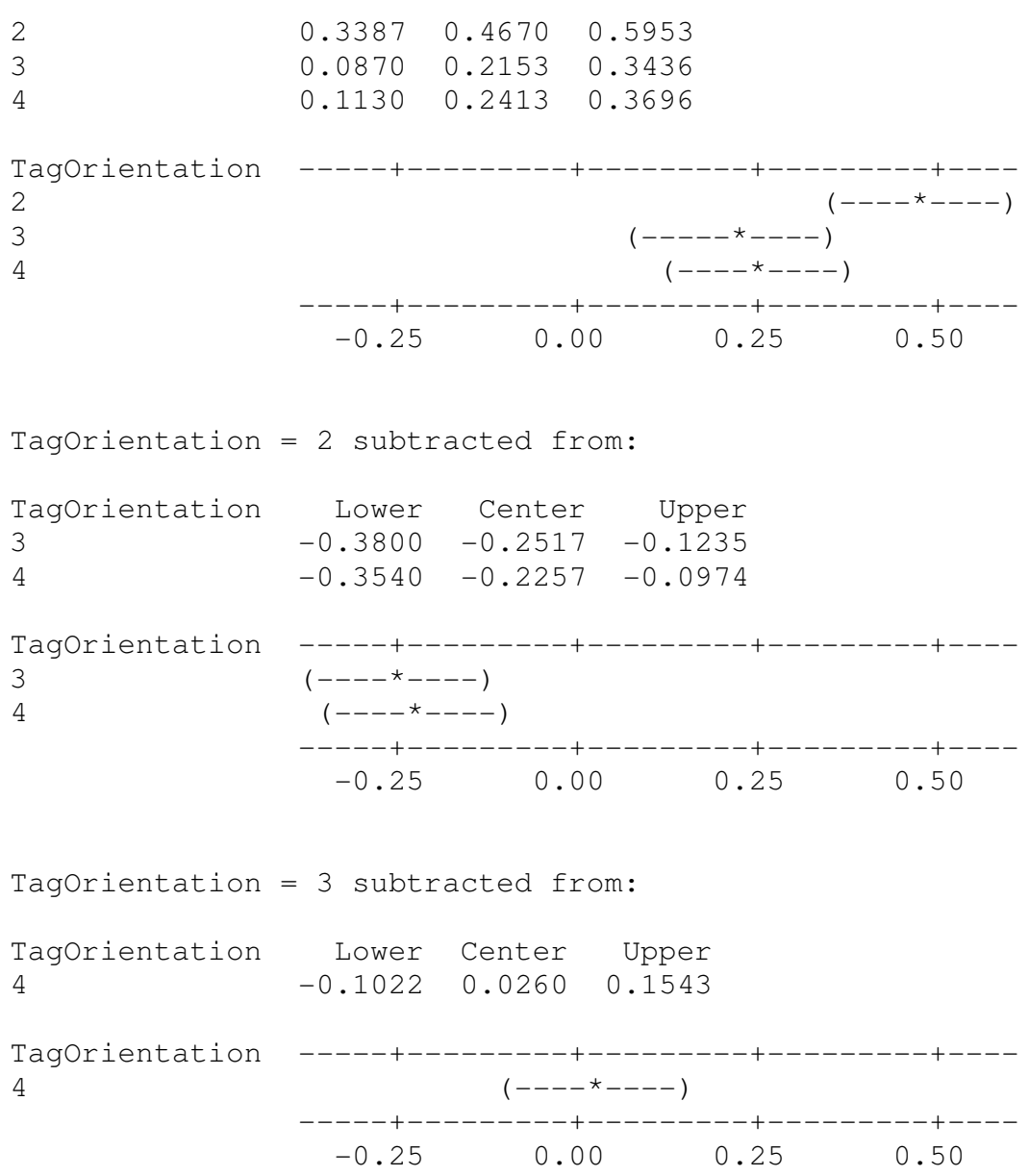

\section{Significance of Scan Method}

Levels of Scan Method

1: Stationary (80s)

2: Antenna Rotation (80s)

3: Tag Board Rotation (80s)

\section{One-way ANOVA: RR versus Scan Method}

\begin{tabular}{lrrrrr} 
Source & DF & SS & MS & F & P \\
Scan Method & 2 & 14.763 & 7.382 & 63.82 & 0.000 \\
Error & 573 & 66.273 & 0.116 & & \\
Total & 575 & 81.036 & & \\
S $=0.3401$ & R-Sq $=18.22 \%$ & R-Sq(adj) $=17.93 \%$ \\
& \multicolumn{1}{c}{ Individual 95\% CIs For Mean Based on }
\end{tabular}




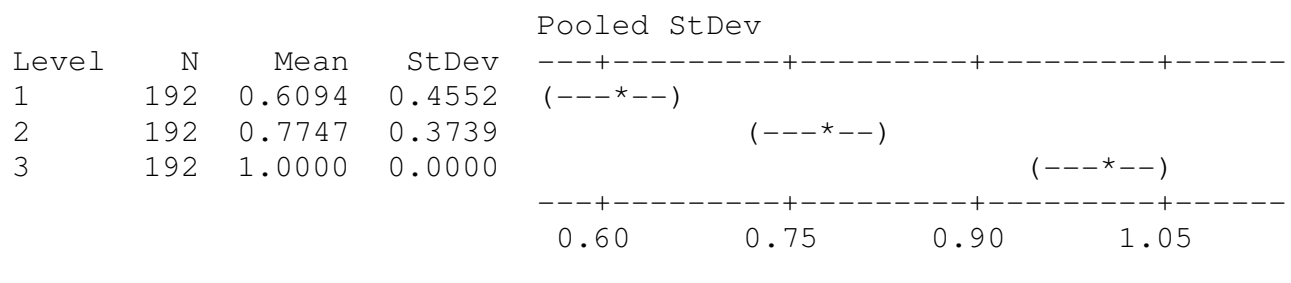

Pooled StDev $=0.3401$

\section{Grouping Information Using Tukey Method}

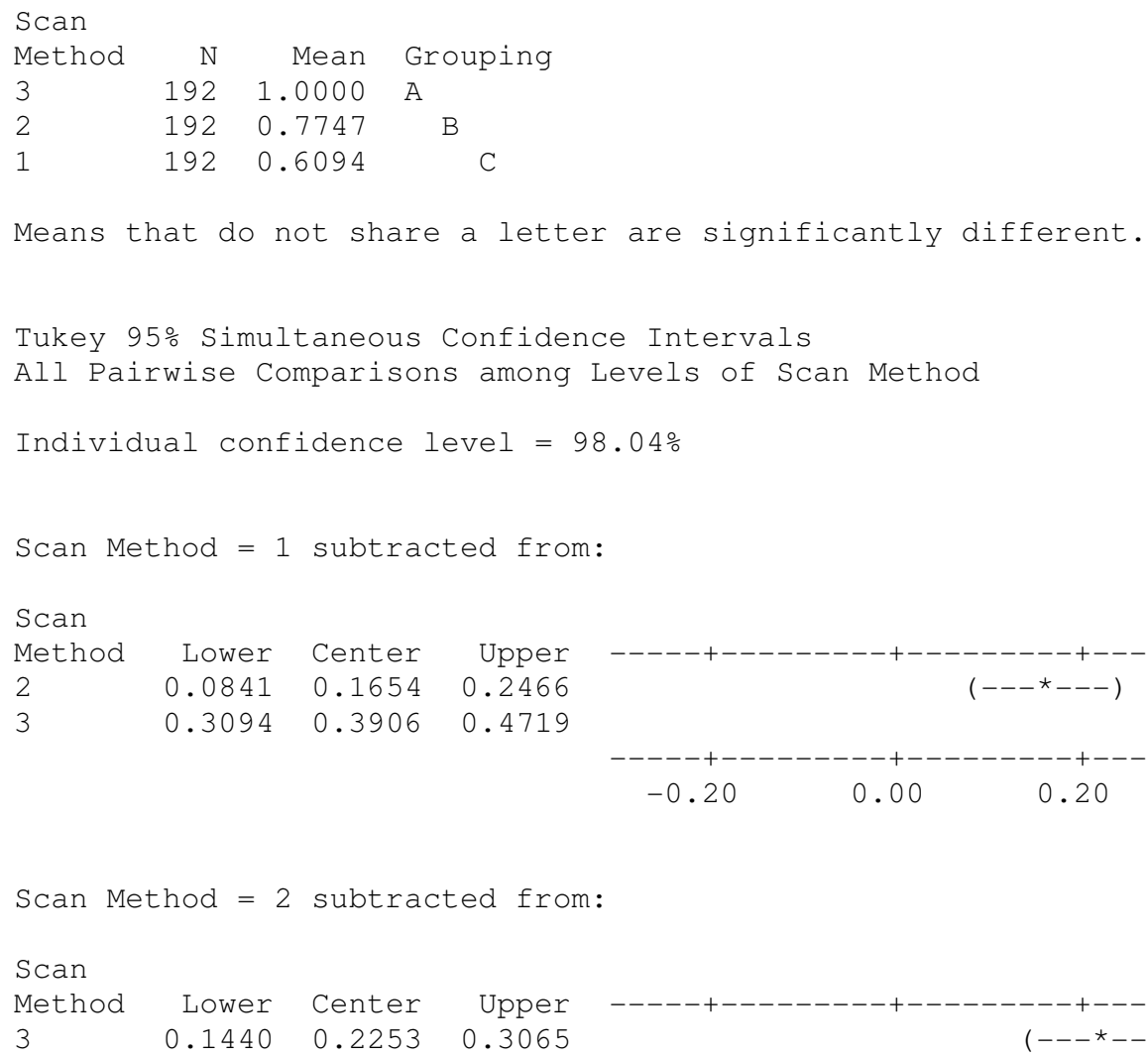

Significance of Light Fixture Interference

\section{Paired T-Test and Cl: Light 80s, 80s}

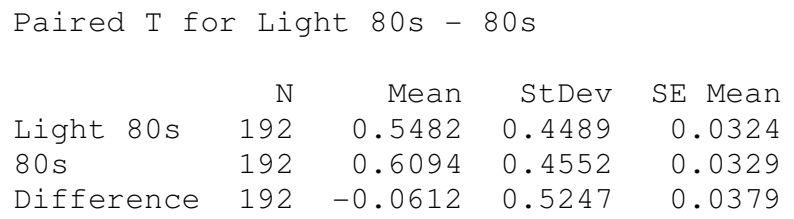


95\% upper bound for mean difference: 0.0014

$\mathrm{T}$-Test of mean difference $=0(\mathrm{vs}<0):$ T-Value $=-1.62 \quad$ P-Value $=0.054$

\section{Significance of Read Duration}

Paired T-Test and $\mathrm{Cl}: 80 \mathrm{~s}, 180 \mathrm{~s}$

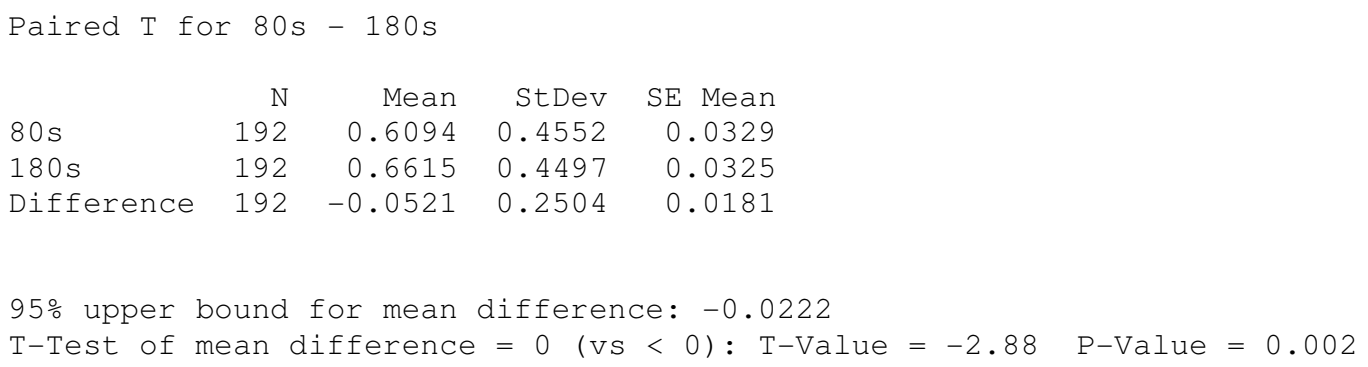




\title{
Appendix B: Experiment Data and Results
}

\author{
Testing Notes
}

\begin{tabular}{|c|c|c|c|c|c|}
\hline $\begin{array}{l}\text { Test } \\
\text { Name }\end{array}$ & $\begin{array}{l}\text { Ant } \\
\text { Pos. }\end{array}$ & $\begin{array}{l}\text { Scan } \\
\text { Method }\end{array}$ & $\begin{array}{l}\text { Read } \\
\text { Duration }\end{array}$ & $\begin{array}{l}\text { Tags } \\
\text { Read }\end{array}$ & Notes \\
\hline $\begin{array}{l}\text { Light } \\
80 \text { s.A }\end{array}$ & 1 & Stationary & $1: 20$ & 101 & $\begin{array}{l}\text { First position of clockwise rotation. Upper left: A, B, } \\
\text { C, D }\end{array}$ \\
\hline $\begin{array}{l}\text { Light } \\
\text { 80s.D }\end{array}$ & 1 & Stationary & $1: 20$ & 106 & $\begin{array}{l}\text { Second position in clockwise rotation. Upper left: D, } \\
\text { A, B, C }\end{array}$ \\
\hline $\begin{array}{l}\text { Light } \\
\text { 80s.C }\end{array}$ & 1 & Stationary & $1: 20$ & 102 & Third position. Upper left: C, D, A, B \\
\hline $\begin{array}{l}\text { Light } \\
80 \text { s.B }\end{array}$ & 1 & Stationary & $1: 20$ & 112 & Fourth position. Upper left: B, C, D, A \\
\hline 80s.A & 2 & Stationary & $1: 20$ & 120 & \multirow{4}{*}{$\begin{array}{l}\text { Moved antenna two feet ( } 1 \text { ceiling panel) away from } \\
\text { nearest light fixture, testing to see if light fixture } \\
\text { effects tag readability. First position (A upper left } \\
\text { corner). Antenna Position } 2 \text { is this new position. } 45 \\
\text { degree shows a } 92 \% \text { in } 6 x 6 \text {, finally some good results }\end{array}$} \\
\hline 80s.D & 2 & Stationary & $1: 20$ & 113 & \\
\hline 80s.C & 2 & Stationary & $1: 20$ & 116 & \\
\hline 80s.B & 2 & Stationary & $1: 20$ & 119 & \\
\hline 180s.A & 2 & Stationary & 3:00 & 124 & \multirow{4}{*}{$\begin{array}{l}\text { Test } 180 \text { s will change the read duration } \\
\text { (run time) from 1:20 minutes to } 3: 00 \\
\text { minutes to test if there is a significant } \\
\text { effect on the read rate. }\end{array}$} \\
\hline 180s.D & 2 & Stationary & 3:00 & 128 & \\
\hline 180s.C & 2 & Stationary & 3:00 & 125 & \\
\hline 180s.B & 2 & Stationary & 3:00 & 131 & \\
\hline $\begin{array}{l}\text { Tag } \\
360.1\end{array}$ & 2 & Tag 360 & $1: 20$ & 192 & \multirow{4}{*}{$\begin{array}{l}\text { The cardboard pieces are secured together with tape, } \\
\text { and string is attached to two opposing corners. A pen } \\
\text { is secured to the tables directly beneath the center of } \\
\text { the antenna. It is stuck through the point at which all } \\
\text { four cardboard pieces meet. The strings are held by } \\
\text { two assistants, and used to rotate the cardboard piece } \\
360 \text { degrees over a 1:20 time span at an approximate } \\
\text { constant velocity. Every } 20 \text { seconds, the cardboard } \\
\text { piece rotates } 90 \text { degrees. }\end{array}$} \\
\hline $\begin{array}{c}\text { Tag } \\
360.2\end{array}$ & 2 & Tag 360 & $1: 20$ & 192 & \\
\hline $\begin{array}{c}\text { Tag } \\
360.3\end{array}$ & 2 & Tag 360 & $1: 20$ & 192 & \\
\hline $\begin{array}{l}\text { Tag } \\
360.4\end{array}$ & 2 & Tag 360 & $1: 20$ & 192 & \\
\hline $\begin{array}{c}\text { Ant } \\
360.1 \\
\end{array}$ & 2 & $\begin{array}{c}\text { Antenna } \\
360\end{array}$ & $1: 20$ & 149 & \multirow{4}{*}{$\begin{array}{l}\text { Installed Lazy Susan; rotating antenna manually for } \\
1: 20 \text { read duration to see effects of scan method. }\end{array}$} \\
\hline $\begin{array}{c}\text { Ant } \\
360.2\end{array}$ & 2 & $\begin{array}{l}\text { Antenna } \\
360\end{array}$ & $1: 20$ & 149 & \\
\hline $\begin{array}{c}\text { Ant } \\
360.3 \\
\end{array}$ & 2 & $\begin{array}{c}\text { Antenna } \\
360\end{array}$ & $1: 20$ & 150 & \\
\hline $\begin{array}{c}\text { Ant } \\
360.4\end{array}$ & 2 & $\begin{array}{c}\text { Antenna } \\
360\end{array}$ & $1: 20$ & 147 & \\
\hline
\end{tabular}




\section{Appendix C: Program Code}

\section{User Interface}

Option Compare Database

Option Explicit

Private WithEvents sockRecv As Winsock

Private WithEvents sockmain As Winsock

Dim strData As String

Dim antenna As Single

Dim testnum As String

Dim all_ants(3)

Private Sub cmdRefresh_Click()

Dim x As Integer

For $\mathrm{x}=1$ To 100

Me.Controls.Item("name" \& x).Visible = False

Me.Controls.Item("pic" \& x). Visible = False

Next

Call locate_tags

End Sub

Private Sub cmdSRefresh_Click()

'antenna $=0$

'prepare tables

With DoCmd

.SetWarnings False

.OpenQuery "purgelocation", acViewNormal, acAdd

.OpenQuery "SendToLocation", acViewNormal, acAdd

.SetWarnings True

End With

'testnum = InputBox("Please enter a test name", "Test Name")

"gather data

"Call Sirit_send("modem.antennas.perform_check()")

'all_ants $(0)=1$

'all_ants $(1)=2$

'all_ants(2) $=3$

'all_ants(3) = 4 
'Call Sirit_send("antennas.mux_sequence=1 23 4")

'Call Sirit_send("tag.reporting.report_fields=tag_id antenna")

'Call Sirit_send("reader.events.register(id=" \& Val(Me.txtSessionID) \& ",name=event.tag.report)")

'Call Sirit_send("setup.operating_mode=active")

'this is the amount of time in miliseconds that each antenna will read for

'Form. TimerInterval $=4000$

Dim x As Integer

For $\mathrm{x}=1$ To 100

Me.Controls.Item("name" \& x).Visible = False

Me.Controls.Item("pic" \& x).Visible $=$ False

Next

DoEvents

Call locate_tags

End Sub

Private Sub Form_Load()

antenna $=0$

Call locate_tags

Call Sirit_connect

End Sub

Sub locate_tags ()

On Error GoTo ender

Dim str1 As String

Dim zone As Single, ZoneLeft As Single, ZoneTop As Single

Dim x As Integer, y As Integer

Dim ZoneQty(9) 'because there are 9 zones - starts at 0

For $\mathrm{x}=0$ To 9

'starts counting at 0

ZoneQty $(\mathrm{x})=0$

Next

Dim rst As ADODB.Recordset, rst1 As ADODB.Recordset

Set rst $=$ New ADODB.Recordset

Set rst1 = New ADODB.Recordset

rst1.Open "SELECT DISTINCT TagID FROM UniqueLocations", _ 
CurrentProject.Connection, adOpenStatic, adLockOptimistic

If rst1.RecordCount $=0$ Then Exit Sub

rst1.MoveFirst

For $\mathrm{y}=1$ To rst1.RecordCount

rst.Open "SELECT StudentInfo.Picture, UniqueLocations.Antenna, [fname] \& "" "" \& [lname] AS FullName " \& _

"FROM UniqueLocations INNER JOIN StudentInfo ON UniqueLocations. TagID = StudentInfo.TagID " \&

"WHERE StudentInfo.TagID = "'" \& rst1("TagID") \& "'" ORDER BY

UniqueLocations.Antenna", _

CurrentProject.Connection, adOpenStatic, adLockOptimistic

If rst.RecordCount $=0$ Then GoTo ender

rst.MoveFirst

zone $=0$

$\operatorname{str} 1=" "$

For $\mathrm{x}=1$ To rst.RecordCount

str1 = str1 \& "," \& rst("antenna")

rst.MoveNext

Next

$\operatorname{str} 1=\operatorname{Right}(\operatorname{str} 1, \operatorname{Len}(\operatorname{str} 1)-1)$

Select Case str1

Case "1"

zone $=1$

ZoneLeft $=0$ 'the left side of zone 1

ZoneTop $=0$ 'the top of zone 1

Case "2"

zone $=3$

ZoneLeft $=6$ 'the left of zone 2

ZoneTop $=0$ 'the top of zone 2

Case "1,2"

zone $=2$

ZoneLeft $=3$

ZoneTop $=0$

End Select

If zone $=0$ Then

zone $=9$

ZoneLeft $=4$

ZoneTop $=0$ 


\section{End If}

rst.MoveFirst

With Me.Controls("pic" \& y)

.Picture = CurrentProject.Path \& "lpicture directoryl" \& rst("Picture")

'1440 is the number of twips...it's a conversion between pixels and inches

'the 4 corresponds to the number of pictures across

.Left $=$ ZoneLeft $* 1440+1440 * 3 *($ ZoneQty(zone $) / 3-\operatorname{Int}($ ZoneQty $($ zone $) / 3))$

'1.3 is the height of the picture+the height of the name label

'the 4 corresponds to the number of pictures across

.Top $=$ ZoneTop $* 1.3 * 1440+1.3 * 1440 * \operatorname{Int}($ ZoneQty(zone) $/ 3)$

.Visible $=$ True

End With

With Me.Controls("name" \& y)

.Caption $=$ rst ("FullName")

' 1440 is the number of twips...it's a conversion between pixels and inches

'the 4 corresponds to the number of pictures across

. Left $=$ ZoneLeft $* 1440+1440 * 3 *($ ZoneQty $($ zone $) / 3-\operatorname{Int}($ ZoneQty $($ zone $) / 3))$

'1.3 is the height of the picture+the height of the name label

'the 4 corresponds to the number of pictures across

.Top $=$ ZoneTop $* 1.3 * 1440+1.3 * 1440 * \operatorname{Int}($ ZoneQty(zone) $/ 3)+1 * 1440$

. Visible $=$ True

End With

'increases the count of members in the zone - it starts counting at 0

ZoneQty (zone $)=$ ZoneQty $($ zone $)+1$

ender:

rst1.MoveNext

rst.Close

Next

End Sub

Private Sub Form_Timer()

If antenna $<$ UBound(all_ants) Then

Call Sirit_send("antennas.mux_sequence=" \& all_ants(antenna))

antenna $=$ antenna +1

Else 
Call Sirit_send("setup.operating_mode=standby")

Me.Form. TimerInterval $=0$

DoCmd.OpenQuery "UniqueLocations", acViewNormal

'DoCmd.OpenForm "ZoneTracker", acNormal

End If

End Sub

Private Sub pic_Click()

Dim z As Integer

Dim a As Integer

$\mathrm{Z}=$ picnumber

If Me.SelectStudentImage. Visible $=$ True Then

Me.SelectStudentImage. Visible $=$ False

End If

If Me.Controls.Item("pic" \& z).BorderStyle = 2 Then

Me.Controls.Item("pic" \& z).BorderStyle $=0$

Me.SelectStudentImage. Visible $=$ False

Else

Me.Controls.Item("pic" \& z).BorderStyle $=2$

Me.Controls.Item("pic" \& z).BorderColor $=5753088$

Me.Controls.Item("pic" \& z).BorderWidth $=3$

Me.SelectStudentImage. Visible $=$ True

End If

Me.SelectStudentImage.Picture = Me.Controls.Item("pic" \& z).Picture

End Sub

Private Sub sockRecv_DataArrival(ByVal bytesTotal As Long)

Call sockRecv.GetData(strData)

If Me.txtSessionID = "" Or IsNull(Me.txtSessionID) Then

If Left $($ strData, 22) $=$ "event.connection id $=$ " Then

Me.txtSessionID = Right(strData, Len(strData) - 22)

End If

Exit Sub

End If

Call loc_data

End Sub

Sub Sirit_connect()

Me.txtSessionID = "" 
Set sockmain $=$ New Winsock

Set sockRecv $=$ New Winsock

With sockmain

.RemoteHost = DLookup("host", "sirit_settings")

.RemotePort = DLookup("portSend", "sirit_settings")

.Connect

End With

With sockRecv

.RemoteHost = DLookup("host", "sirit_settings")

.RemotePort = DLookup("portRecv", "sirit_settings")

.Connect

End With

End Sub

Sub Sirit_send(strCommand As String)

sockmain.SendData strCommand \& $\mathrm{Chr}(10)$

End Sub

Sub loc_data()

Dim TagAry As Variant

Dim x As Integer

Dim rst As ADODB.Recordset, rst1 As ADODB.Recordset

Set rst $=$ New ADODB . Recordset

Set rst $1=$ New ADODB.Recordset

rst.Open "[location]", CurrentProject.Connection, adOpenStatic, adLockOptimistic

Dim fields(2)

fields $(0)=$ "TagID"

fields $(1)=$ "Antenna"

fields $(2)=$ "TestNumber"

Dim values(2)

TagAry = Split(strData, "event.tag.report tag_id=0x")

If IsArray (TagAry) $=$ False Then Exit Sub

For $\mathrm{x}=1$ To UBound(TagAry)

values $(0)=\operatorname{Left}(\operatorname{Tag} \operatorname{Ary}(\mathrm{x}), 24)$

$\operatorname{values}(1)=\operatorname{Val}(\operatorname{Mid}(\operatorname{Tag} A r y(x), 35,1))$

values $(2)=$ testnum

If Len $($ values $(0))=24$ And Not $(\operatorname{values}(1)=0)$ Then rst.AddNew fields, values Next

rst.Close

End Sub 


\section{Reader Command}

Option Compare Database

Option Explicit

Private WithEvents sockmain As Winsock

Private WithEvents sockRecv As Winsock

Dim ActiveMode As Boolean, KeepAlive As Boolean, SkipData As Boolean, andy As

Boolean

Dim strData As String

Dim antenna As Single

Dim all_ants(0)

'Dim all_ants(3) is for multiple antennas

Dim testnum As String

Private Sub cboSend_AfterUpdate()

If Len(Me.cboSend) > 0 Then Me.txtArgs = DLookup("arguments", "sirit_commands", "command = """ \& Me.cboSend \& """" )

End Sub

Private Sub cmdActiveMode_Click()

Me.txtStatus = ""

If Me.cmdActiveMode.Caption = "Active mode $=$ off" Then

Me.cmdActiveMode. Caption $=$ "Active mode $=$ on"

Call Sirit_send("tag.reporting.report_fields=tag_id antenna time")

Call Sirit_send("reader.events.register(id=" \& Val(Me.txtSessionID) \& ",name=event.tag.report)")

Call Sirit_send("setup.operating_mode=active")

ActiveMode $=$ True

GoTo ending

End If

If Me.cmdActiveMode. Caption = "Active mode $=$ on" Then

Me.cmdActiveMode.Caption = "Active mode=off"

Call Sirit_send("reader.events.unregister(id=" \& Val(Me.txtSessionID) \&

",name=event.tag.report)")

Call Sirit_send("setup.operating_mode=standby")

ActiveMode $=$ False

End If 
GoTo ending

ending:

DoEvents

End Sub

Private Sub cmdClear_Click()

Me.txtStatus = ""

End Sub

Private Sub cmdConnect_Click()

Call Sirit_connect

Me.txtSessionID = ""

KeepAlive $=$ True

Me.Form. TimerInterval $=3000$

End Sub

Private Sub cmdScanLoc_Click()

antenna $=0$

'prepare tables

With DoCmd

.SetWarnings False

.OpenQuery "SendToHistory", acViewNormal, acAdd

.OpenQuery "purgelocation", acViewNormal, acAdd

.SetWarnings True

End With

testnum $=$ InputBox("Please enter a test name", "Test Name")

andy $=$ True

'gather data

'Call Sirit_send("modem.antennas.perform_check()")

all_ants $(0)=1$

'all_ants $(1)=2$

'all_ants $(2)=3$

'all_ants $(3)=4$

Call Sirit_send("antennas.mux_sequence=1")

Call Sirit_send("tag.reporting.report_fields=tag_id antenna")

Call Sirit_send("reader.events.register(id=" \& Val(Me.txtSessionID) \&

",name=event.tag.report)")

Call Sirit_send("setup.operating_mode=active")

Form. TimerInterval $=$ Form.runlength.Value $* 1000$

End Sub 


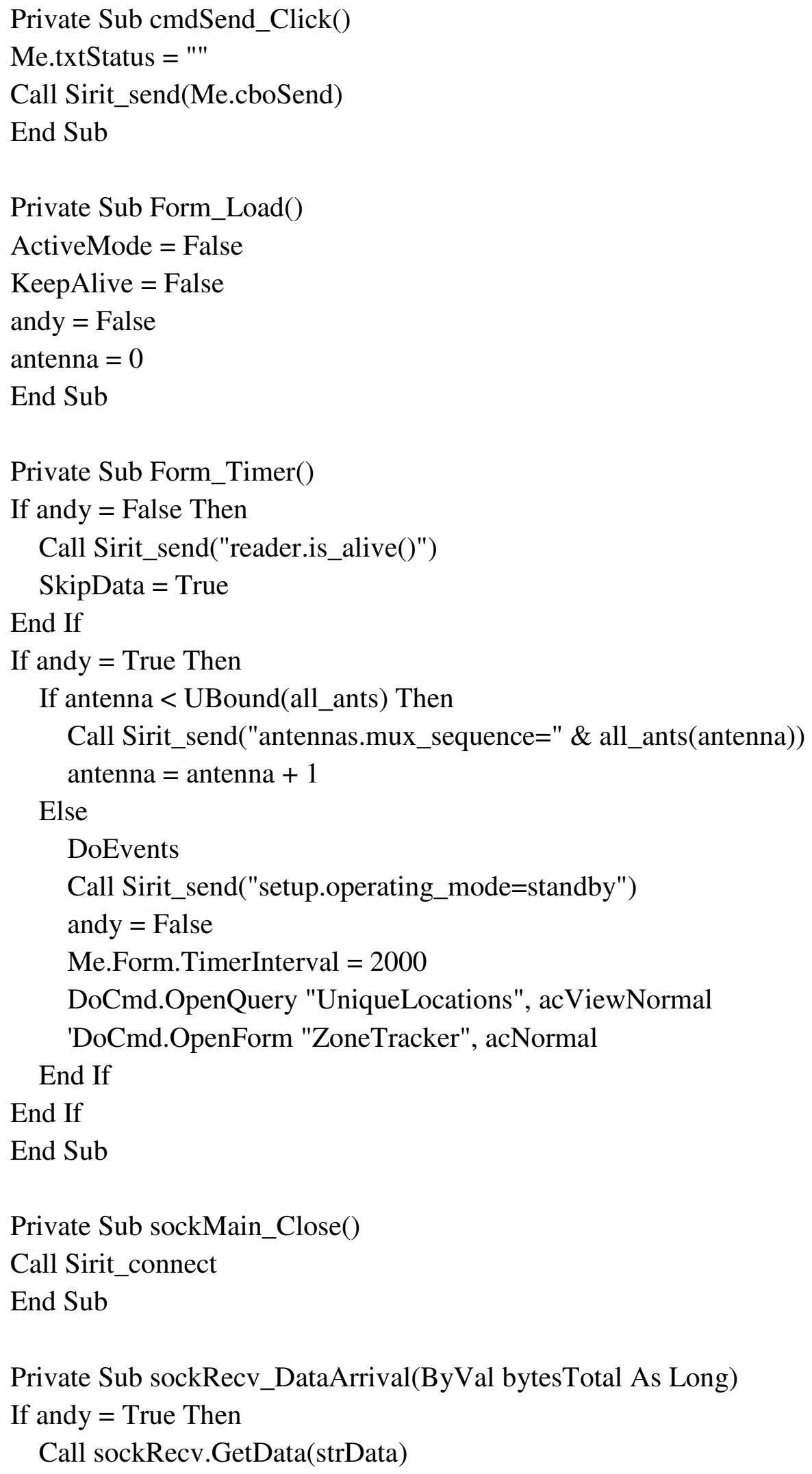


Call loc_data

Exit Sub

End If

Call sockRecv.GetData(strData)

If ActiveMode $=$ True Then Call add_data

If ActiveMode $=$ False Then

If Left $($ strData, 22) $=$ "event.connection id $=$ " Then Me.txtSessionID = Right $($ strData, Len(strData) - 22)

End If

End Sub

Private Sub sockmain_DataArrival(ByVal bytesTotal As Long)

If andy $=$ True Then

Call sockmain.GetData(strData)

'all_ants = Split(Right(strData, Len(strData) - 3), " ")

'Debug.Print Right(strData, Len(strData) - 2)

End If

If SkipData $=$ True Then

SkipData $=$ False

Call sockmain.GetData(strData)

Exit Sub

End If

Call sockmain.GetData(strData)

Me.txtStatus $=$ strData

End Sub

Sub Sirit_connect()

Me.txtSessionID = ""

Set sockmain $=$ New Winsock

Set sockRecv $=$ New Winsock

With sockmain

.RemoteHost = DLookup("host", "sirit_settings")

.RemotePort = DLookup ("portSend", "sirit_settings")

.Connect

End With

With sockRecv 


$$
\begin{aligned}
& . \text { RemoteHost = DLookup("host", "sirit_settings") } \\
& \text {.RemotePort = DLookup("portRecv", "sirit_settings") } \\
& \text {.Connect }
\end{aligned}
$$

End With

End Sub

Sub Sirit_send(strCommand As String)

sockmain.SendData strCommand \& $\operatorname{Chr}(10)$

End Sub

Sub loc_data()

Dim TagAry As Variant

Dim x As Integer

Dim rst As ADODB.Recordset, rst1 As ADODB.Recordset

Set rst $=$ New ADODB . Recordset

Set rst $1=$ New ADODB.Recordset

rst.Open "[location]", CurrentProject.Connection, adOpenStatic, adLockOptimistic Dim fields(2)

$$
\begin{aligned}
& \text { fields }(0)=\text { "TagID" } \\
& \text { fields }(1)=\text { "Antenna" } \\
& \text { fields }(2)=\text { "TestNumber" }
\end{aligned}
$$

Dim values(2)

TagAry = Split(strData, "event.tag.report tag_id=0x")

If IsArray (TagAry) $=$ False Then Exit Sub

For $\mathrm{x}=1$ To UBound(TagAry)

$$
\begin{aligned}
& \text { values( }(0)=\operatorname{Left}(\operatorname{Tag} \operatorname{Ary}(\mathrm{x}), 24) \\
& \text { values }(1)=\operatorname{Val}(\operatorname{Mid}(\operatorname{Tag} \operatorname{Ary}(\mathrm{x}), 35,1)) \\
& \text { values }(2)=\operatorname{testnum}
\end{aligned}
$$

If Len $(\operatorname{values}(0))=24$ And Not $(\operatorname{values}(1)=0)$ Then rst.AddNew fields, values

Next

rst.Close

End Sub

Sub add_data()

Dim TagAry As Variant

Dim x As Integer

Dim rst As ADODB.Recordset, rst1 As ADODB.Recordset

Set rst $=$ New ADODB.Recordset

Set rst $1=$ New ADODB.Recordset

rst.Open "[read]", CurrentProject.Connection, adOpenStatic, adLockOptimistic 
Dim fields(2)

$$
\begin{aligned}
& \text { fields }(0)=\text { "TagID" } \\
& \text { fields }(1)=\text { "TimeRead" } \\
& \text { fields }(2)=\text { "Antenna" }
\end{aligned}
$$

Dim values(2)

TagAry = Split(strData, "event.tag.report tag_id=0x")

If IsArray(TagAry) = False Then Exit Sub

For $\mathrm{x}=1$ To UBound(TagAry)

$$
\begin{aligned}
& \text { values }(0)=\operatorname{Left}(\operatorname{Tag} \operatorname{Ary}(x), 24) \\
& \text { values }(1)=\operatorname{Mid}(\operatorname{Tag} \operatorname{Ary}(x), 54,12) \& \text { " " \& Date } \\
& \text { values(2) }=\operatorname{Mid}(\operatorname{Tag} \operatorname{Ary}(x), 35,1)
\end{aligned}
$$

If Len $(\operatorname{values}(0))=24$ And Len $(\operatorname{values}(1))>0$ And $\operatorname{Not}(\operatorname{Left}(\operatorname{values}(2), 1)=$ " ") = True Then

rst1.Open "SELECT DISTINCT * FROM [Read] WHERE TagID = "'" \& values(0)

\& """", CurrentProject.Connection, adOpenStatic, adLockOptimistic

If rst1.RecordCount $=0$ Then

rst.AddNew fields, values

rst1.Close

Else

rst1.Close

rst1.Open "UPDATE [Read] SET [Read].TimeRead = "'" \& values(1) \& ""'

WHERE $($ Read.TagID $)=$ "'" \& values $(0) \&$ ""'""

End If

End If

Next

rst.Close

End Sub 